\title{
Una contribución al entendimiento sobre el cultivo en fases del langostino Macrobrachium rosenbergii en Costa Rica
}

\author{
A contribution to the understanding of the culture in phases of the freshwater \\ prawn Macrobrachium rosenbergii in Costa Rica
}

\author{
José Valverde ${ }^{1,2}$
}

\section{Resumen}

\begin{abstract}
El objetivo del presente estudio fue contribuir al entendimiento de los efectos del cultivo en fases sobre la productividad y el cambio compensatorio del langostino Macrobrachium rosenbergii con el fin de aumentar la intensidad del cultivo y manejar el crecimiento heterogéneo por medio de la separación de tallas. El estudio se llevó a cabo en una finca acuícola en la región Caribe de Costa Rica. Se utilizó una pileta de concreto de $12 \mathrm{~m}^{2} \times 0.5 \mathrm{~m}$ de profundidad como precría y dos estanques de tierra cubiertos con plástico de $72 \mathrm{~m}^{2} \times 0.8 \mathrm{~m}$ de hondo en las fases de desarrollo y engorde. La pileta de precría fue dividida en dos y las densidades de siembra fueron de $91.6 / \mathrm{m}^{2}$ por sección. En precría se cosecharon con $2.5 \mathrm{~g}$ promedio para sembrar el estanque de desarrollo dividido en dos a una densidad de $12.5 / \mathrm{m}^{2}$ por sección. En desarrollo se volvieron a cosechar con 7.3 g promedio (1.3-19.5 g). Se separaron por tamaños por medio de una red y los grandes se sembraron con peso promedio de $10.4 \mathrm{~g}$ en un estanque a $6.1 / \mathrm{m}^{2}$ y los pequeños de $4.1 \mathrm{~g}$ promedio en otro a $4.8 / \mathrm{m}^{2}$. Las producciones fueron de $191.1 \mathrm{~g} / \mathrm{m}^{2}$ en precría, $79.9 \mathrm{~g} / \mathrm{m}^{2}$ en desarrollo y $77.6 \mathrm{~g} / \mathrm{m}^{2}$ en engorde. Los índices del rendimiento productivo indicaron escaso avance con el traslado de la precría al desarrollo y engorde bajando las densidades de siembra, excepto cuando fueron separados por tamaños, donde los pequeños incrementaron considerablemente la biomasa inicial (2.7 veces) y contribuyeron al $50 \%$ de la producción en engorde. El promedio de la productividad fue de $1128 \mathrm{~kg} / \mathrm{ha} / \mathrm{año}$,
\end{abstract}

\footnotetext{
${ }^{1}$ Instituto Nacional de Aprendizaje, Núcleo Náutico Pesquero, Puntarenas, Costa Rica

${ }^{2}$ E-mail: jvalverdemoya@ina.ac.cr
}

Recibido: 11 de enero de 2021

Aceptado para publicación: 15 de junio de 2021

Publicado: 24 de agosto de 2021

CLos autores. Este artículo es publicado por la Rev Inv Vet Perú de la Facultad de Medicina Veterinaria, Universidad Nacional Mayor de San Marcos. Este es un artículo de acceso abierto, distribuido bajo los términos de la licencia Creative Commons Atribución 4.0 Internacional (CC BY 4.0) [https:// creativecommons.org/licenses/by/4.0/deed.es] que permite el uso, distribución y reproducción en cualquier medio, siempre que la obra original sea debidamente citada de su fuente original 
pero como el cultivo en fases permite hacer hasta 3.5 ciclos de engorde al año, la productividad se puede incrementar a $2716 \mathrm{~kg} / \mathrm{ha} / \mathrm{año}$. Al separar los langostinos por tamaño para sembrarlos en el engorde se notó crecimiento compensatorio en los pertenecientes a la fracción de los grandes dentro de un amplio rango de variabilidad de tallas. El porcentaje del peso de la cola disminuyó con el aumento en el tamaño del animal y fue mayor para las hembras que para los machos debido al menor tamaño y peso de sus tenazas. Los resultados preliminares de este estudio indican que el cultivo en fases puede aumentar la productividad al poder realizarse mayor cantidad de ciclos al año y la separación de tallas para mejorar el tamaño a cosecha de los langostinos pequeños.

Palabras clave: langostinos, Macrobrachium rosenbergii, densidad de siembra, crecimiento compensatorio, productividad

\section{Abstract}

The aim of this study was to contribute to the understanding of the effects of phased cultivation on the productivity and the compensatory change of Macrobrachium rosenbergii prawns to increase the intensity of the cultivation and manage the heterogeneous growth through size separation. The study was carried out in an aquaculture farm in the Caribben region of Costa Rica. A concrete pool measuring $12 \mathrm{~m}^{2}$ $\mathrm{x} 0.5 \mathrm{~m}$ deep was used as nursery and two earthen ponds covered with plastic measuring $72 \mathrm{~m}^{2} \times 0.8 \mathrm{~m}$ deep for the development and fattening phases. The nursery pool was divided in two and the stocking densities were $91.6 / \mathrm{m}^{2}$ per section. In the nursery they were harvested with an average $2.5 \mathrm{~g}$ to stock the development pond divided in two at a density of $12.5 / \mathrm{m}^{2}$ per section. In development they were harvested again with an average $7.3 \mathrm{~g}$ (1.3-19.5 g). They were separated by size using a net and the large ones were transferred with an average weight of $10.4 \mathrm{~g}$ in one pond at $6.1 / \mathrm{m}^{2}$ and the small ones with an average $4.1 \mathrm{~g}$ in another at $4.8 / \mathrm{m}^{2}$. The productions were $191.1 \mathrm{~g} / \mathrm{m}^{2}$ in nursery, $79.9 \mathrm{~g} / \mathrm{m}^{2}$ in development and $77.6 \mathrm{~g} / \mathrm{m}^{2}$ in fattening. The productive yield indices indicated slow progress with the transfer from nursery to development and fattening, lowering the stocking densities, except when they were separated by size, where the small ones considerably increased the initial biomass (2.7 times) and contributed to $50 \%$ of the fattening production. The average productivity was $1128 \mathrm{~kg} / \mathrm{ha} / \mathrm{year}$, but since phased cultivation allows up to 3.5 fattening cycles per year, productivity can be increased to $2716 \mathrm{~kg} / \mathrm{ha} /$ year. When separating the prawns by size to stock them in the fattening, compensatory growth was noted in those belonging to the fraction of the large ones within a wide range of size variability. The percentage of the weight of the tail decreased with the increase in the size of the animal and was greater for the females than for the males due to the smaller size and weight of their claws. The preliminary results of this study indicate that cultivation in phases can increase productivity by being able to carry out more cycles per year and the separation of sizes to improve the crop size of small prawns.

Key words: prawns, Macrobrachium rosenbergii, planting density, compensatory growth, productivity 


\section{INTRODUCCIÓN}

La sostenibilidad del cultivo de langostinos en aguas territoriales con bajas densidades de siembra y fertilización orgánica para maximizar la productividad natural ha sido demostrada (New et al., 2000). El langostino Macrobrachium rosenbergii es una especie candidata para promover un cultivo acuícola rural sostenible operado por núcleos familiares como fuente de ingresos para las familias y comunidades locales. Las oportunidades existen en Costa Rica para invertir en este tipo de proyectos como alternativa al declive de las pesquerías de camarón y los problemas de enfermedades en sus cultivos (New, 2000a). El cultivo de langostinos en el país alcanzó su máxima expresión en 1999 después de haberse dado un aumento considerable desde 1993, pero ya se predecía la decadencia de su cultivo desde que Langostinos KoKo cerró sus operaciones a nivel de finca, a pesar de que mantuvo el vivero capaz de producir 12 millones de pos-larvas (PL's) al año y existía un mercado local fuerte vendiendo al pie de finca o con distribuidores (New, 2000b).

La falta de investigación y desarrollo, tanto en Costa Rica como en los demás países centroamericanos, sobre el cultivo de langostinos ha provocado que su recuperación sea muy lenta. La mayoría de los artículos científicos se han publicado en regiones templadas (Valenti y New, 2000), necesitándose de estudios en los trópicos sobre el efecto de la densidad de siembra y la separación de tallas de los juveniles sobre los rendimientos de producción y la rentabilidad del cultivo. El primer caso fue estudiado por Valverde y Varela (2020) usando dos densidades de siembra (2.5 y 6.0 langostinos $\left./ \mathrm{m}^{2}\right)$ en estanques. Se determinó que la productividad fue superior en la densidad de siembra más alta, mientras que la rentabilidad solo se presentó en la densidad de siembra más baja debido a que los costos de producción fueron más bajos por la compra de menor cantidad de semilla y los ingresos por ventas en granja fueron los más altos debido a la mayor proporción de organismos con talla comercial a mejor precio de mercado.

Con respecto al efecto de la separación de tallas, se hace necesaria la implementación del cultivo en fases para el traslado de los juveniles. Esta fue una técnica recomendada desde 1981 con el fin de reemplazar el sistema tradicional de siembra directa y cosechas parciales o totales (Alston y Sampaio, 2000). El sistema de monocultivo tradicional presenta varias desventajas como el manejo ineficiente del crecimiento heterogéneo individual entre hembras y machos, caracterizados por los morfo tipos de machos en grandes (MG) de tenazas azules, medianos (MM) de tenazas anaranjadas y pequeños (MP) de tenazas cortas y claras (Holthuis, 2000). Además, se tiene un ineficiente y laborioso trabajo en las cosechas selectivas, alta mortalidad de las poslarvas recién sembradas, excesivo tiempo de residencia y menor aprovechamiento de la capacidad de carga de los estanques (Karplus et al., 2000).

Debido a estas y otras razones técnicas y de mercado, muchos estudios han demostrado que la tecnología de monocultivo tradicional no es rentable en relativamente altas densidades de siembra (Rhodes, 2000; Valverde y Varela, 2020). A pesar de ello, este sistema semi-intensivo de cultivo con alimentación suplementaria es el más comúnmente utilizado a nivel mundial desde 1992 (Rhodes, 2000). Se han alcanzado producciones de 1000 a $3000 \mathrm{~kg} / \mathrm{ha} / \mathrm{año}$ dentro de un rango de 500 a $5000 \mathrm{~kg} / \mathrm{ha} / \mathrm{año}$, pero con un manejo muy simple y lejos de explorar todo su potencial (Valenti y New, 2000).

La tecnología del cultivo en fases la implementó de manera comercial la empresa Sabana Grande en Puerto Rico de 1991 a 1998. La técnica consiste básicamente en hacer transferencias entre tanques y estanques reduciendo las densidades de siembra y seleccionando por tamaños (Alston y Sampaio, 2000). La primera fase es la maternidad que se da en el vivero después de 
aclimatar las PL's (300 PL/g) al agua dulce (2-3 días) y pasarlas a tanques en alta densidad (de 200 hasta $2000 \mathrm{PL} / \mathrm{m}^{3}$ ) donde permanecen de 20 a 60 días hasta alcanzar el estadio juvenil I (0.1-0.5 g). Luego se transportan a las unidades productivas donde se siembran en la fase de precría (PC), ya sea en piletas, encierros, jaulas o estanques pequeños. Se siembran desde 75 hasta $1500 / \mathrm{m}^{2}$ y se cosechan al cabo de 4-10 semanas con un peso cercano a $2.0 \mathrm{~g}$ (juveniles II). A esta edad ya muestran alto crecimiento heterogéneo individual (se nota desde que son PL's en el vivero), por lo que en zonas templadas se separan en grupos aumentando la productividad y rentabilidad (Tidwell y D'Abramo, 2000). En zonas tropicales, el efecto de separarlos por tamaños debe ser investigado (Valenti y New, 2000). Finalmente se pasan a la fase de desarrollo reduciendo la densidad de siembra a $20-30 / \mathrm{m}^{2}$ hasta que alcanzan un peso de 9-15 g al cabo de 60-90 días, cuando se cosechan y trasladan a los estanques de engorde hasta talla comercial. La separación por tamaños de la fase de desarrollo a la de engorde no ha sido documentada.

En engorde se reduce la densidad de siembra desde $10 / \mathrm{m}^{2}$ hasta $4-5 / \mathrm{m}^{2}$ según el diseño y el manejo de la unidad productiva en regiones tropicales. Se debe considerar que conforme aumenta la densidad de siembra se incrementa la producción, pero se reduce el tamaño del producto (Valenti y New, 2000; Valverde y Varela, 2020). La duración de la fase de engorde depende del momento en que se alcanza el tamaño comercial, usualmente arriba de $30 \mathrm{~g}$ (Tidwell y D'Abramo, 2000).

Por lo tanto, el cultivo en fases constituye una tecnología apropiada para manejar el crecimiento heterogéneo y aumentar la intensidad del cultivo por medio de la separación de tallas, técnica que ha tenido un profundo y persistente efecto en la población de machos. El morfo tipo de los MP es reversible, al contrario de los otros dos, dando cambio compensatorio a MM y MG (Karplus et al., 2000). El efecto de la separación de tallas sobre la productividad del cultivo de langostinos debe ser establecido en zonas tropicales como la forma de manejar el crecimiento heterogéneo individual en relativamente altas densidades de siembra. El objetivo de estudio fue contribuir al entendimiento de los efectos del cultivo en fases sobre la productividad y el cambio compensatorio del langostino Macrobrachium rosenbergii en Costa Rica.

\section{Materiales y Métodos}

El estudio se llevó a cabo del 28 de febrero al 21 de noviembre de 2020 en una finca acuícola ubicada en el distrito La Rita $\left(10^{\circ} 12^{\prime} 56^{\prime \prime} \mathrm{N}\right.$ y $\left.83^{\circ} 47^{\prime} 32^{\prime \prime} \mathrm{O}\right)$ del cantón de Pococí, en la región Caribe de la provincia de Limón, Costa Rica. Se utilizó una pileta de concreto de $12 \mathrm{~m}^{2} \times 0.5 \mathrm{~m}$ de profundidad como precría (PC) y dos estanques de tierra cubiertos con plástico de $72 \mathrm{~m}^{2} \times 0.8 \mathrm{~m}$ de hondo cada uno para las fases de desarrollo (D) y engorde (E).

Se sembraron 1100 juveniles I en PC después de haber pasado un periodo de maternidad de 30 días en tanques dentro del vivero donde alcanzaron un peso promedio de $0.1 \mathrm{~g}$. Se aclimataron por 30 minutos a la temperatura existente en la pileta de cemento. Esta fue forrada con plástico negro y dividida a la mitad con una red de malla mosquetero prensada en el fondo. Las densidades de siembra fueron de $91.6 / \mathrm{m}^{2}\left(550 \mathrm{PL} / 6 \mathrm{~m}^{2}\right)$ en cada pileta (PC1 y PC2). Durante este periodo se cubrieron con una malla para evitar el ataque de aves. El agua se mantuvo con aireación constante utilizando una pequeña bomba sumergible llamada fuente de poder con el fin de que la lanzara agua en forma de chorro en cada división. Se mantuvieron refugios formados por tejas para techo en igual cantidad por pileta. Aquí permanecieron por 70 días cuando se cosecharon como juveniles II para trasladarlos a la fase de desarrollo. 
En la fase de desarrollo se sembraron juveniles II de 35 a $80 \mathrm{~mm}$ y de 0.5 a $6.9 \mathrm{~g}$ $(50.0 \pm 0.8 \mathrm{~mm}$ y $2.5 \pm 0.2 \mathrm{~g}$, respectivamente), dentro de un rango de 0.7 a $7.0 \mathrm{~g}$. La siembra fue en un estanque de tierra de $72 \mathrm{~m}^{2}$ forrado con plástico negro y dividido a la mitad por una red de malla mosquetero prensada en el fondo. Cada división (D1 y D2) fue provista con una fuente de poder para la aireación. Se sembraron a una densidad de $12.5 / \mathrm{m}^{2}$ (449 juveniles/ $36 \mathrm{~m}^{2}$ ) en cada estanque.

En ese estanque permanecieron por 83 días cuando se cosecharon los pre-adultos para trasladarlos a la fase de engorde. En la cosecha se obtuvieron pre-adultos de 40 a $95 \mathrm{~mm}$ y de 1.3 a $19.5 \mathrm{~g}(68.0 \pm 1.0 \mathrm{~mm}$ y 7.3 $\pm 0.1 \mathrm{~g}$, respectivamente). Aprovechando la disparidad de tallas, se separaron grandes de pequeños por medio de una red puesta en un tanque con agua y con un hueco de malla que solo permitía pasar a los más pequeños. Una muestra $(\mathrm{n}=30)$ de cada grupo fue medida. Los langostinos grandes $(10.4 \pm 0.8 \mathrm{~g})$ se sembraron en un nuevo estanque (E1) de $72 \mathrm{~m}^{2}$ forrado con plástico negro y los langostinos pequeños $(4.1 \pm 0.5 \mathrm{~g})$ se volvieron a sembrar en el mismo estanque (E2) donde se cosecharon de desarrollo, después de un lavado del fondo. Las densidades utilizadas fueron de $6.1 / \mathrm{m}^{2}$ (438 langostinos $/ 72 \mathrm{~m}^{2}$ ) para los grandes y $4.8 / \mathrm{m}^{2}$ (348 langostinos $/ 72 \mathrm{~m}^{2}$ ) para los pequeños.

En los estanque E1 y E2 permanecieron durante 83 y 113 días, respectivamente cuando se realizó la cosecha final. Se mantuvieron estructuras piramidales hechas de tubos PVC de 3" como refugios en igual cantidad por estanque. La aireación fue constante con las fuentes de poder y no se protegieron con mallas de las aves. Los langostinos se cosecharon por medio de un vaciado de ambos estanques por gravedad y con el uso de una motobomba eléctrica para sacar los remanentes de agua en las fosas de cosecha. Se contabilizaron y se les dio una muerte rápida por medio de un choque térmico en agua con hielo. Se analizó una muestra de un mínimo de 100 individuos elegidos al azar, midiendo la longitud ( $\mathrm{mm}$ ) total y de las tenazas, así como el peso (g) total, de la cabeza, las tenazas y el abdomen para cada individuo según sexo y morfo tipo.

Se hicieron muestreos quincenales en todas las fases para determinar el crecimiento y observar a simple vista el estado de salud. Para esto, se capturaron al azar 30 individuos para pesarlos en una balanza electrónica Ohaus $( \pm 0.05 \mathrm{~g})$ y medirlos (desde el borde anterior del ojo hasta la punta del telson) con una regla graduada en $\mathrm{mm}( \pm 0.5 \mathrm{~mm})$. Después se regresaron al respectivo aposento productivo. Posterior a la siembra de los langostinos en cada fase, se procedió a medir en ambos recintos productivos de manera diaria el oxígeno disuelto y la temperatura del agua en las mañanas (06:00) a $10 \mathrm{~cm}$ del fondo utilizando el dispositivo Hanna HI9146 y en dos días de cada semana la turbidez del agua a las 10:00 con un disco Secchi.

Las pérdidas por evaporación se repusieron introduciendo agua de un pozo artesanal tipo cisterna. Cuando el agua se ponía turbia y de coloración verde $(<20 \mathrm{~cm})$, se hacían recambios hasta mantener una turbidez de $30-40 \mathrm{~cm}$, medida con el disco Sechii. Los recambios de agua fueron superficiales y leves $(5 \%)$ y solo se incrementaron en casos de emergencia para reponer niveles bajos de oxígeno disuelto (OD).

La alimentación se inició a partir del segundo día en precría y se mantuvo durante todo el periodo de estudio. Se proporcionó Biomar ${ }^{\circledR}(35 \%$ de proteína) dos veces al día según la tabla de alimentación (Cuadro 1) y la observación de residuos de alimento en el fondo del recinto productivo. Si sobraba alimento, se suspendía la alimentación por 1-2 días hasta que lo consumieran todo. Debe tomarse en cuenta que en las piletas y estanques de cultivo y muy notablemente en los de tierra, se genera una importante cantidad de microalgas bentónicas y planctónicas, además de los microorganismos que se alimentan de ellas, y que les sirven a los langostinos como alimento natural que forma parte del 
Cuadro 1. Tabla de alimentación utilizada en el estudio

\begin{tabular}{cc}
\hline $\begin{array}{c}\text { Rango de peso } \\
(\mathrm{g})\end{array}$ & $\begin{array}{c}\text { Porcentaje del } \\
\text { peso }\end{array}$ \\
\hline $0.006-0.5$ & 5.00 \\
$0.5-1.0$ & 4.50 \\
$1.0-1.5$ & 4.00 \\
$1.5-3.0$ & 3.00 \\
$3.0-4.5$ & 2.00 \\
$4.5-6.0$ & 1.50 \\
$6.0-7.5$ & 1.25 \\
$7.5-10.0$ & 1.00 \\
$10.0-15.0$ & 0.75 \\
$>15.0$ & 0.50 \\
\hline
\end{tabular}

detritus presente. El aporte de este alimento no fue tomado en cuenta para el cálculo de la conversión alimenticia en cada recinto productivo.

Las condiciones experimentales entre las fases fueron las densidades de siembra altas en precría $\left(91.6 / \mathrm{m}^{2}\right)$, medianas en desarrollo $\left(12.5 / \mathrm{m}^{2}\right)$ y bajas en engorde $\left(6.1 / \mathrm{m}^{2}\right.$ para grandes y $4.8 / \mathrm{m}^{2}$ para los pequeños). Los rendimientos de producción (promedios \pm desviación estándar) se obtuvieron según procedimientos establecidos por Valverde y Varela (2020) con los organismos cosechados en cada fase. La producción en $\mathrm{kg} / \mathrm{ha} /$ año se estimó multiplicando el número de ciclos por los $\mathrm{kg} / \mathrm{ha}$ y el rendimiento se calculó con los kilogramos producidos por cada 1000 juveniles (millar) sembrados.

El índice del tamaño de la producción (ITP) combina la producción total $\left(\mathrm{g} / \mathrm{m}^{2}\right)$ con el peso promedio final $(\mathrm{g}) \mathrm{y}$ da una medida de la habilidad del sistema para aumentar la producción mientras mantiene el peso. Se obtuvo por la fórmula: ITP $=\mathrm{P} \times \mathrm{Pf} / 100$, donde: $\mathrm{P}=$ Producción; $\mathrm{Pf}=$ Peso promedio fi- nal. La conversión alimenticia (CA) se calculó dividiendo la cantidad de alimento aplicado entre la ganancia de biomasa en cada fase de cultivo: $\mathrm{CA}=\mathrm{C} / \mathrm{Bf}-\mathrm{Bi}$, donde: $\mathrm{C}=$ cantidad de alimento; $\mathrm{Bf}$ y $\mathrm{Bi}=$ Biomasa final y biomasa inicial.

La demostración de la existencia del crecimiento compensatorio y su efecto sobre la producción del cultivo se hizo comparando los promedios \pm desviación estándar de la tasa relativa de crecimiento semanal (TRC) y la tasa específica de crecimiento (TEC). Ambas se obtuvieron con las fórmulas:

- $\quad$ TRC $=$ Pf - Pi / Pi, donde: Pf y Pi = Peso final y peso inicial en cada fase de cultivo.

- $\quad$ TEC $(\%$ aumento en peso $(\% \mathrm{~g} /$ día $))=$ [(ln Pf - ln Pi) / t] x 100, donde: $\ln$ Pf y ln $\mathrm{Pi}=$ logaritmo natural del peso final $\mathrm{e}$ inicial, $\mathrm{t}=$ tiempo en días

No se realizó el análisis estadístico de los datos obtenidos debido al bajo número de tratamientos con sus respectivas réplicas. Se trata, por lo tanto, de resultados preliminares obtenidos de piletas y estanques a nivel comercial que requieren mayor investigación en regiones tropicales, pero que aun así muestran similitud con resultados obtenidos en otras latitudes.

\section{Resultados}

\section{Calidad del Agua}

Las variaciones mensuales de la temperatura, el OD y la turbidez del agua medidos en las mañanas en los recintos experimentales durante las fases de cultivo se muestran en el Cuadro 2. El OD presentó los valores más bajos en las mañanas $(1.8 . \pm 0.6 \mathrm{mg} / \mathrm{l})$ en octubre 2020 durante la fase de engorde, llegándose a presentar mortalidad en el estanque con los langostinos grandes después de un periodo de muda fuerte. Inmediatamente se hizo recambio de agua y se puso aireación de emergencia llegándose a restablecer 
Cuadro 2. Valores promedio ( \pm desviación estándar - D.E.) de parámetros fisicoquímicos del agua medidos en las mañanas durante los meses de estudio en las fases de cultivo del langostino Macrobrachium rosenbergii (Costa Rica)

\begin{tabular}{llcccccc}
\hline \multirow{2}{*}{ Mes } & Fases & \multicolumn{2}{c}{$\begin{array}{c}\text { Oxígeno disuelto } \\
(\mathrm{mg} / \mathrm{l})\end{array}$} & \multicolumn{2}{c}{$\begin{array}{c}\text { Temperatura } \\
\left({ }^{\circ} \mathrm{C}\right)\end{array}$} & \multicolumn{2}{c}{$\begin{array}{c}\text { Disco Sechii } \\
(\mathrm{cm})\end{array}$} \\
\cline { 3 - 8 } & & Prom. & D.E. & Prom. & D.E. & Prom. & D.E. \\
\hline Mar & Precría & 4.8 & 0.8 & 29.2 & 0.4 & 45.1 & 3.5 \\
Abr & Precría & 2.7 & 0.5 & 28.8 & 1.7 & 40.6 & 1.4 \\
May & Desarrollo & 2.5 & 0.1 & 28.7 & 1.6 & 41.4 & 7.1 \\
Jun & Desarrollo & 3.2 & 0.1 & 28.6 & 1.4 & 40.0 & 6.4 \\
Jul & Desarrollo & 2.4 & 0.2 & 28.5 & 0.7 & 38.8 & 2.8 \\
Ag & Engorde & 2.5 & 0.4 & 28.9 & 1.3 & 44.9 & 3.5 \\
Set & Engorde & 4.3 & 0.5 & 29.3 & 1.8 & 40.5 & 8.5 \\
Oct & Engorde & 1.8 & 0.6 & 28.8 & 0.4 & 34.0 & 4.2 \\
Nov & Engorde & 3.7 & 1.3 & 28.3 & 0.9 & 38.0 & 1.7 \\
\hline
\end{tabular}

las condiciones al día siguiente. En los restantes meses no hubo problemas por falta de $\mathrm{OD}$, con valores arriba de $2.0 \mathrm{mg} / \mathrm{l}$. La temperatura del agua presentó valores relativamente constantes en las mañanas. El agua fue más clara cada vez que se iniciaba una fase de cultivo, pero con el paso de los días se fue tornando más turbia. El nivel más oscuro $(34.0 \pm 4.2 \mathrm{~cm})$ se dio en octubre cuando se presentaron los problemas de OD en uno de los estanques de engorde.

\section{Problemas de Salud}

En los muestreos quincenales en la fase de engorde se notó la aparición de manchas negras en el caparazón de los langostinos, sobre todo en las zonas cercanas a las branquias (Figura 1). La afectación fue más evidente en el estanque con los langostinos grandes, donde se pudo observar la caída de gran cantidad de hojas provenientes de los árboles y plantas cercanos. Para su correc- ción, se procedió a la aplicación semanal de $40 \mathrm{~g}$ de cal al estanque durante los dos últimos meses de cultivo. El tratamiento también se hizo en el estanque con los langostinos pequeños, aunque estaban menos afectados por la necrosis del caparazón.

A pesar de que las condiciones mejoraron con el tratamiento con cal debido a que se redujo la cantidad de manchas negras en el caparazón, en un día del mes de octubre, con mudas fuertes y niveles bajos de oxígeno disuelto $(1.0 \mathrm{mg} / \mathrm{l})$, se dio una alta mortalidad de langostinos. Todos los animales muertos se extrajeron manualmente del fondo del estanque ( $\mathrm{n}=50$ individuos). Los que permanecieron vivos se notaban oscuros y débiles. Se procedió a hacer un fuerte recambio diario de agua hasta que los niveles de oxígeno disuelto fueron los adecuados $(>3.0 \mathrm{mg} / \mathrm{l})$ al segundo día. Estos langostinos se cosecharon 15 días después de este evento de mortalidad porque ya tenían tamaño de cosecha. 

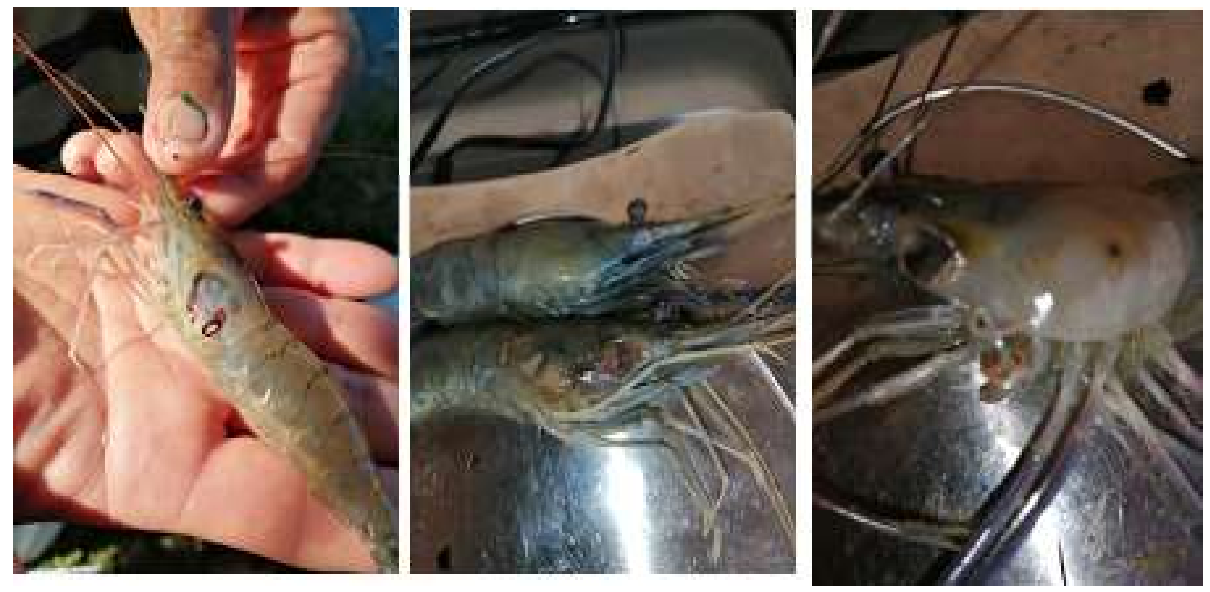

Figura 1. Langostinos con manchas negras y necrosis en el caparazón en la fase de engorde

\section{Rendimientos de Producción}

Los datos del rendimiento de producción en las fases de precría, desarrollo y engorde se presentan en el Cuadro 3. Los pesos finales mostraron poca variación en las fases de precría y desarrollo, mientras que la amplia diferencia de tamaños en la fase de engorde es atribuible a la separación de tallas entre los grandes (10.4 g) en E1 y los pequeños $(4.1 \mathrm{~g})$ en E2 a diferentes densidades de siembra $\left(6.1\right.$ y $4.8 / \mathrm{m}^{2}$, respectivamente). La TRC muestra un valor considerablemente más alto en precría que en las otras dos fases. Los pequeños en engorde mostraron una TRC doblemente superior (2.7) que los sembrados más grandes (1.1). La tasa de sobrevivencia entre las fases estuvo dentro de un rango de 81 a $88 \%$, siendo solo inferior (61\%) en E2 por la mortalidad presentada ante la baja de oxígeno disuelto en agua en un día del cultivo. El crecimiento semanal mostró la relación inversa con respecto a la densidad de siembra, ya que fue de $0.25 \pm$ $0.02 \mathrm{~g} / \mathrm{semana}$ en precría a $91.7 / \mathrm{m}^{2}, 0.62 \pm$ $0.01 \mathrm{~g} / \mathrm{semana}$ en desarrollo a $12.5 / \mathrm{m}^{2}$ y 1.40 $\pm 0.64 \mathrm{~g} / \mathrm{semana}$ a $5.5 / \mathrm{m}^{2}$ como promedio en engorde. Los grandes en E1 crecieron prác- ticamente el doble (1.85 g/semana) que los pequeños en E2 (0.95 g/semana).

La producción en precría fue de 191.1 $\pm 18.2 \mathrm{~g} / \mathrm{m}^{2}$ con una sobrevivencia del $82 \%$ y un peso final de $2.5 \mathrm{~g}$ al cabo de 70 días de cultivo sembrando juveniles I de $0.1 \mathrm{~g}$. Este valor fue superior al de las otras fases de cultivo $\left(79.9 \pm 9.4 \mathrm{~g} / \mathrm{m}^{2}\right.$ y $77.6 \pm 5.2 \mathrm{~g} / \mathrm{m}^{2}$ en desarrollo y engorde, respectivamente) como una relación directamente proporcional con la mayor densidad de siembra. Sin embargo, no ocurrió descenso en la producción entre desarrollo y engorde a pesar la reducción en la densidad de siembra, probablemente debido al efecto que tuvo la separación de tallas sobre una mayor tasa de crecimiento (de 0.62 \pm 0.01 en desarrollo a $1.40 \pm 0.64 \mathrm{~g} / \mathrm{semana}$ como promedio en engorde). Los respectivos valores del ITP fueron: 4.8 para la precría, 5.8 para el desarrollo y 14.4 para el engorde.

Las biomasas finales muestran un incremento conforme trascurren las fases de cultivo debido a la disminución en las densidades de siembra y la separación de tallas. Con el fin de obviar estos efectos, es importante calcular la biomasa total relativa divi- 
Cuadro 3. Rendimientos de producción en las fases de precría, desarrollo y engorde del langostino Macrobrachium rosenbergii (Costa Rica)

\begin{tabular}{|c|c|c|c|c|c|c|c|c|c|c|c|c|c|c|c|}
\hline \multirow[b]{2}{*}{ Fases } & \multirow{2}{*}{$\frac{\text { Area }}{\left(\mathrm{m}^{2}\right)}$} & \multirow{2}{*}{ Total } & \multirow{2}{*}{$\frac{\text { Dens. }}{\# / \mathrm{m}^{2}}$} & \multirow{2}{*}{ Días } & \multicolumn{2}{|c|}{ Peso (g) } & \multirow[b]{2}{*}{ TRC } & \multirow{2}{*}{$\begin{array}{c}\text { Sobr. } \\
(\%)\end{array}$} & \multirow{2}{*}{$\begin{array}{l}\text { Crecim. } \\
\text { (g/sem) }\end{array}$} & \multicolumn{3}{|c|}{ Biomasa } & \multirow{2}{*}{$\begin{array}{c}\text { - Alim. } \\
\text { (g) }\end{array}$} & \multirow[b]{2}{*}{ C.A. } & \multirow[b]{2}{*}{ T.A. } \\
\hline & & & & & Inicial & Final & & & & $\mathrm{g} / \mathrm{m}^{2}$ & $\begin{array}{c}\text { Final } \\
(\mathrm{g})\end{array}$ & $\begin{array}{l}\text { Inicial } \\
(\mathrm{g})\end{array}$ & & & \\
\hline Pcl & 6 & 550 & 91.7 & 70 & 0.1 & 2.7 & 25.8 & 83 & 0.27 & 203.9 & 1223.4 & 55.0 & 966.9 & 0.8 & 2.3 \\
\hline $\mathrm{Pc} 2$ & 6 & 550 & 91.7 & 70 & 0.1 & 2.4 & 23.0 & 81 & 0.24 & 178.2 & 1069.2 & 55.0 & 744.2 & 0.7 & 1.8 \\
\hline Total & 12 & 1100 & & & & & & & & & 2292.6 & 110.0 & 1711.1 & & \\
\hline Media & & & 91.7 & 70 & 0.1 & 2.5 & 24.4 & 82 & 0.25 & 191.1 & & & & 0.8 & 2.0 \\
\hline D.E. & & & & & & 0.2 & 2.0 & 1 & 0.02 & 18.2 & & & & 0.1 & 0.4 \\
\hline D1 & 36 & 449 & 12.5 & 83 & 2.5 & 7.2 & 1.9 & 96 & 0.61 & 86.6 & 3116.4 & 1122.5 & 2925.5 & 1.5 & 1.0 \\
\hline D2 & 36 & 449 & 12.5 & 83 & 2.5 & 7.4 & 2.0 & 79 & 0.63 & 73.2 & 2635.5 & 1122.5 & 2178.4 & 1.4 & 0.7 \\
\hline Total & 72 & 898 & & & & & & & & & 5751.9 & 2245.0 & 5103.9 & & \\
\hline Media & & & 12.5 & 83 & 2.5 & 7.3 & 1.9 & 88 & 0.62 & 79.9 & & & & 1.5 & 0.9 \\
\hline D.E. & & & & & & 0.1 & 0.1 & 12 & 0.01 & 9.4 & & & & 0.0 & 0.2 \\
\hline E-1 & 72 & 438 & 6.1 & 83 & 10.4 & 21.9 & 1.1 & 61 & 1.85 & 81.3 & 5851.2 & 4555.2 & 2846.7 & 2.2 & 0.5 \\
\hline E-2 & 72 & 348 & 4.8 & 113 & 4.1 & 15.3 & 2.7 & 100 & 0.95 & 74.0 & 5324.4 & 1426.8 & 2537.2 & 0.7 & 0.3 \\
\hline Total & & 786 & & & & & & & & & 11175.6 & 5982.0 & 5383.9 & & \\
\hline Media & & & 5.5 & 98 & 7.3 & 18.6 & 1.9 & 81 & 1.40 & 77.6 & & & & 1.4 & 0.4 \\
\hline D.E. & & & 0.9 & 21 & 4.5 & 4.7 & 1.1 & 28 & 0.64 & 5.2 & & & & 1.1 & 0.1 \\
\hline
\end{tabular}

Dens. $=$ densidad, $T R C=$ Tasa relativa de crecimiento, Sobr.= sobrevivencia, Crecim. $=$ crecimiento, Alim. $=$ alimento, C.A.= Conversión alimenticia, T.A.= Tasa de alimentación (Kg alimento $\left./ \mathrm{m}^{2 *} \mathrm{~d}\right), \mathrm{g} / \mathrm{sem} .=$ gramos por semana, Desvest.=desviación standard

Fechas de siembra: 28 de febrero, 8 de mayo y 4 de agosto para Pc, D y E, respectivamente

diendo la biomasa acumulada entre la biomasa inicial en cada fase. Esta indica el número de veces que se incrementó la biomasa inicial a través del tiempo. En este estudio fue de 20 veces en precría, 1.6 veces en desarrollo y una vez en engorde como promedio, siendo nueve veces mayor en los langostinos pequeños (2.7) que en los grandes (0.3).

En total se produjeron $2.3 \mathrm{~kg}$ en precría, $5.8 \mathrm{~kg}$ en desarrollo y $11.2 \mathrm{~kg}$ en engorde. El rendimiento, medido como la cantidad de kilogramos producidos por cada millar de animales sembrados fue de $2.1,6.5$ y $14.2 \mathrm{~kg}$, respectivamente. De los cosechados en engorde, $52 \%(7.4 \mathrm{~kg})$ correspondió al grupo de los grandes y $48 \%(6.8 \mathrm{~kg})$ al de los pequeños.

La conversión alimenticia fue menor $(0.8: 1.0)$ en precría que en las otras fases (1.4-1.5:1.0), notándose una reducción considerable en E2 (0.7:1.0) donde se sembraron los langostinos pequeños con respecto a E1 con los grandes (2.2:1.0). Una conversión alimenticia inferior a 1 significa que por cada kilogramo de biomasa ganada se invirtió menos de un kilogramo de alimento. La tasa de alimentación fue superior en precría $\left(2.0 \pm 0.4 \mathrm{~g} / \mathrm{m}^{2} / \mathrm{d}\right)$ aplicando del 4 al $5 \%$ de la biomasa en alimento, intermedia en desarro$1 \mathrm{lo}\left(0.9 \pm 0.2 \mathrm{~g} / \mathrm{m}^{2} / \mathrm{d}\right)$ con una aplicación del 1 
Cuadro 4. Cronograma de la producción de langostinos en fases

\begin{tabular}{|c|c|c|c|c|}
\hline Meses & $\begin{array}{c}\text { Precría (Pc1 y } \\
\text { Pc2) }\end{array}$ & $\begin{array}{c}\text { Desarrollo (D1 y } \\
\text { D2) }\end{array}$ & & $\begin{array}{l}\text { Engorde } \\
(\mathrm{E} 1 \mathrm{y} \mathrm{E} 2)\end{array}$ \\
\hline $1-3$ & Siembra A & & & \\
\hline $4-6$ & Siembra B & Desarrollo A & & \\
\hline $7-9$ & Siembra C & Desarrollo B & & \\
\hline $10-12$ & Siembra D & Desarrollo C & & \\
\hline $13-15$ & Siembra E & Desarrollo D & & \\
\hline $16-18$ & Siembra F & Desarrollo E & & \\
\hline $19-21$ & & Desarrollo F & & \\
\hline $7-10$ & & & $1^{\circ}$ año & 1 Engorde A \\
\hline $11-14$ & & & $2^{\circ}$ año & 1 Engorde B \\
\hline $15-18$ & & & & 1 Engorde $\mathrm{C}$ \\
\hline $19-22$ & & & & 1 Engorde D \\
\hline $23-24$ & & & & $1 / 2$ Engorde F \\
\hline
\end{tabular}

al 3\% de la biomasa y baja en engorde $(0.4 \pm$ $0.1 \mathrm{~g} / \mathrm{m}^{2} / \mathrm{d}$ ) cuando se aplicó menos del $1 \%$ de la biomasa.

Una diferencia importante fue la duración de los ciclos de engorde, siendo de 83 días para los langostinos grandes y 113 días para los pequeños. La duración total fue, por lo tanto, de 236 días para los grandes y 266 para los pequeños, con lo cual se pueden hacer 1.5 ciclos por año en el primer caso y 1.4 en el segundo. De tal forma, la productividad sería de $121.9 \mathrm{~g} / \mathrm{m}^{2} /$ año $\left(81.3 \mathrm{~g} / \mathrm{m}^{2}\right.$ en 1.5 ciclos/año) en el primer caso y $103.7 \mathrm{~g} / \mathrm{m}^{2} /$ año $\left(74.0 \mathrm{~g} / \mathrm{m}^{2}\right.$ en 1.4 ciclos/año) en el segundo. El promedio de la productividad se establece en $112.8 \mathrm{~g} / \mathrm{m}^{2} /$ año o $1128 \mathrm{~kg} / \mathrm{ha} /$ año.
La realización del cultivo en fases tiene la ventaja de que mientras los langostinos están en engorde, se puede ir avanzando en las otras fases de precría y desarrollo, con lo cual se pueden hacer más ciclos por año. Se pueden hacer ciclos de 90 días ( 3 meses) en precría y desarrollo considerando una preparación de 20 días en la pileta y 7 días en el estanque. En engorde se consideran ciclos de 120 días (4 meses) quedando de 7 a 37 días para la preparación de los estanques cuando se siembran pequeños y grandes de manera respectiva. Un cronograma de siembras y cosechas en las distintas fases se presenta en el Cuadro 4 donde se separan las fases de precría y desarrollo que duran tres meses y las de engorde que duran cuatro meses. En el cuadro se aprecia que en el pri- 


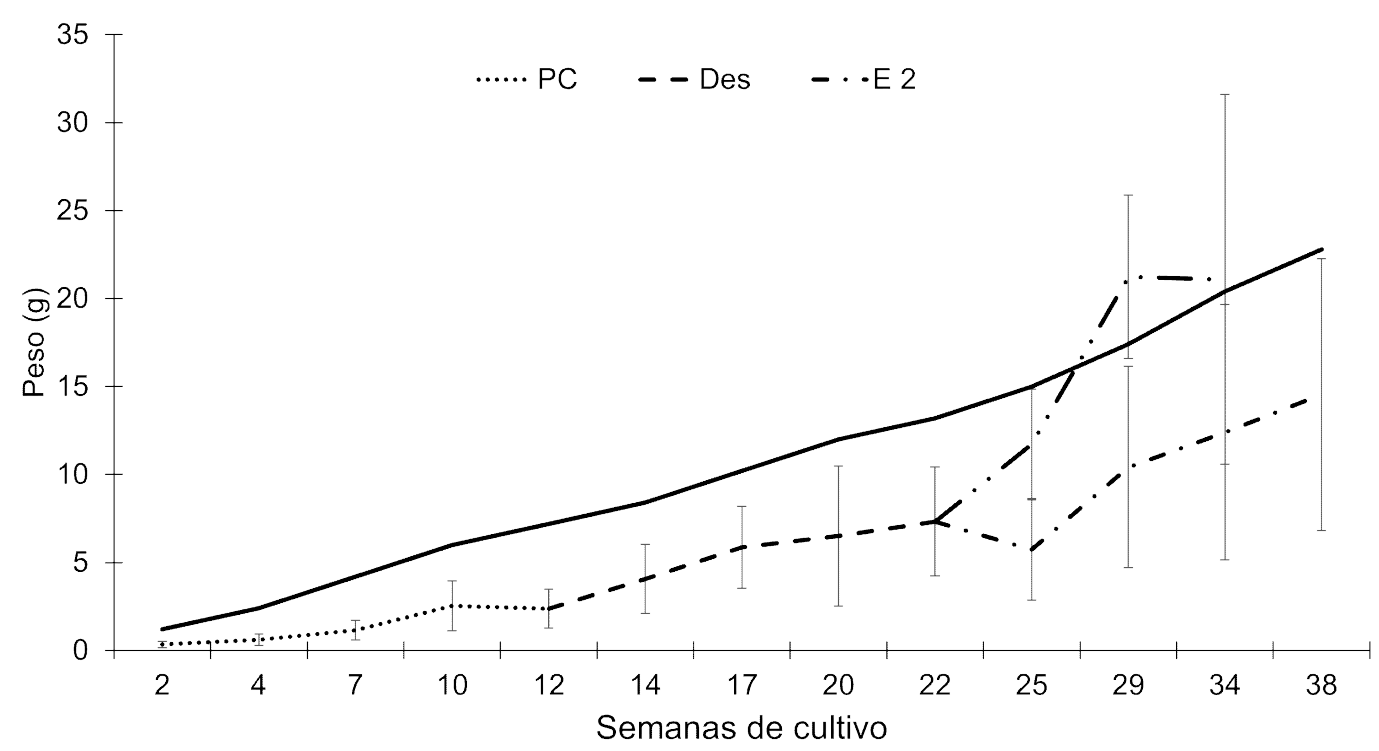

Figura 2. Incremento en peso de langostinos Macrobrachium rosenbergii en las tres fases de cultivo con respecto a un valor teórico con siembra directa en similares densidades (PC: precría; Des: fase de desarrollo; E1: fase de engorde para langostinos de talla grande; E2: fase de engorde para langostinos de talla pequeña)

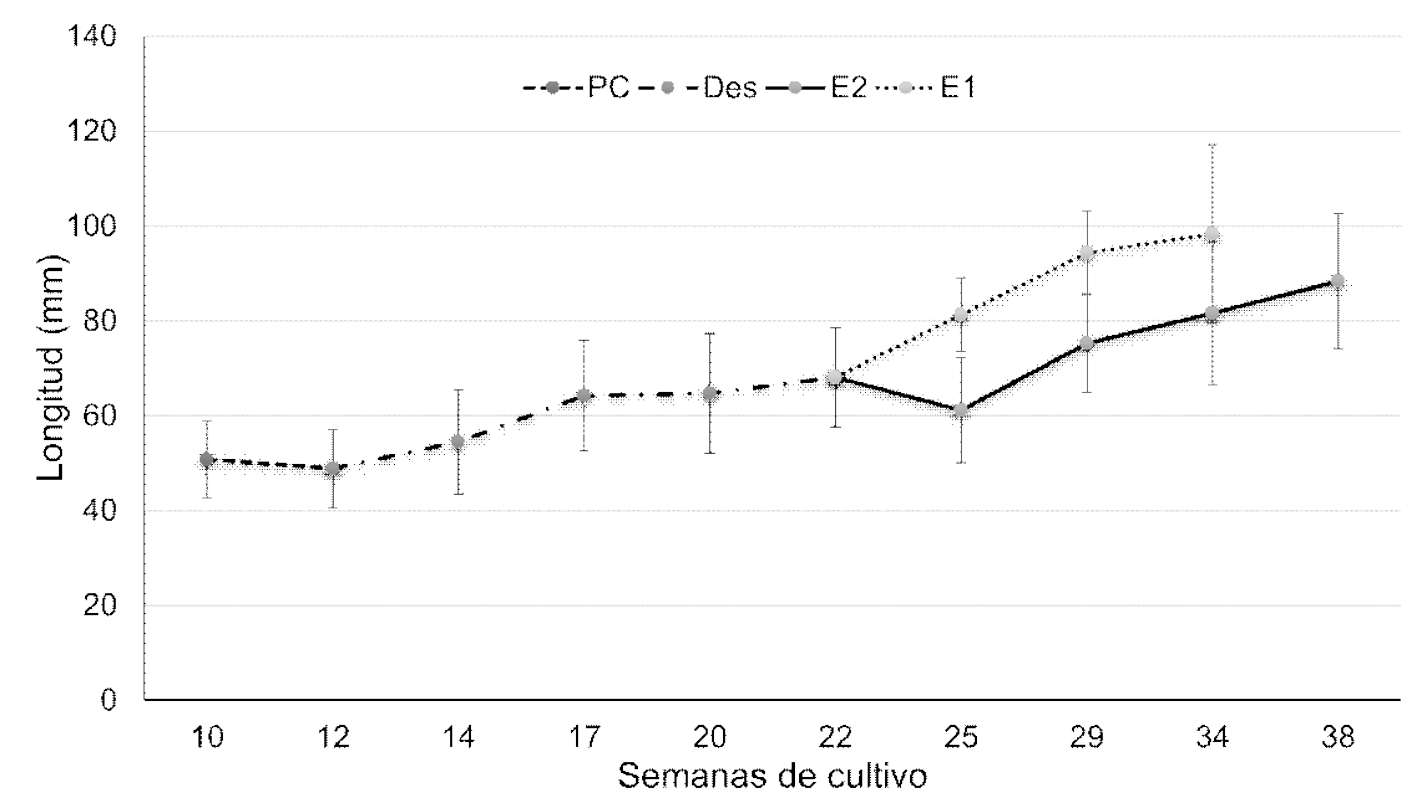

Figura 3. Incremento en longitud de langostinos Macrobrachium rosenbergii en las tres fases de cultivo con respecto a un valor teórico con siembra directa en similares densidades (PC: precría; Des: fase de desarrollo; E1: fase de engorde para langostinos de talla grande; E2: fase de engorde para langostinos de talla pequeña) 


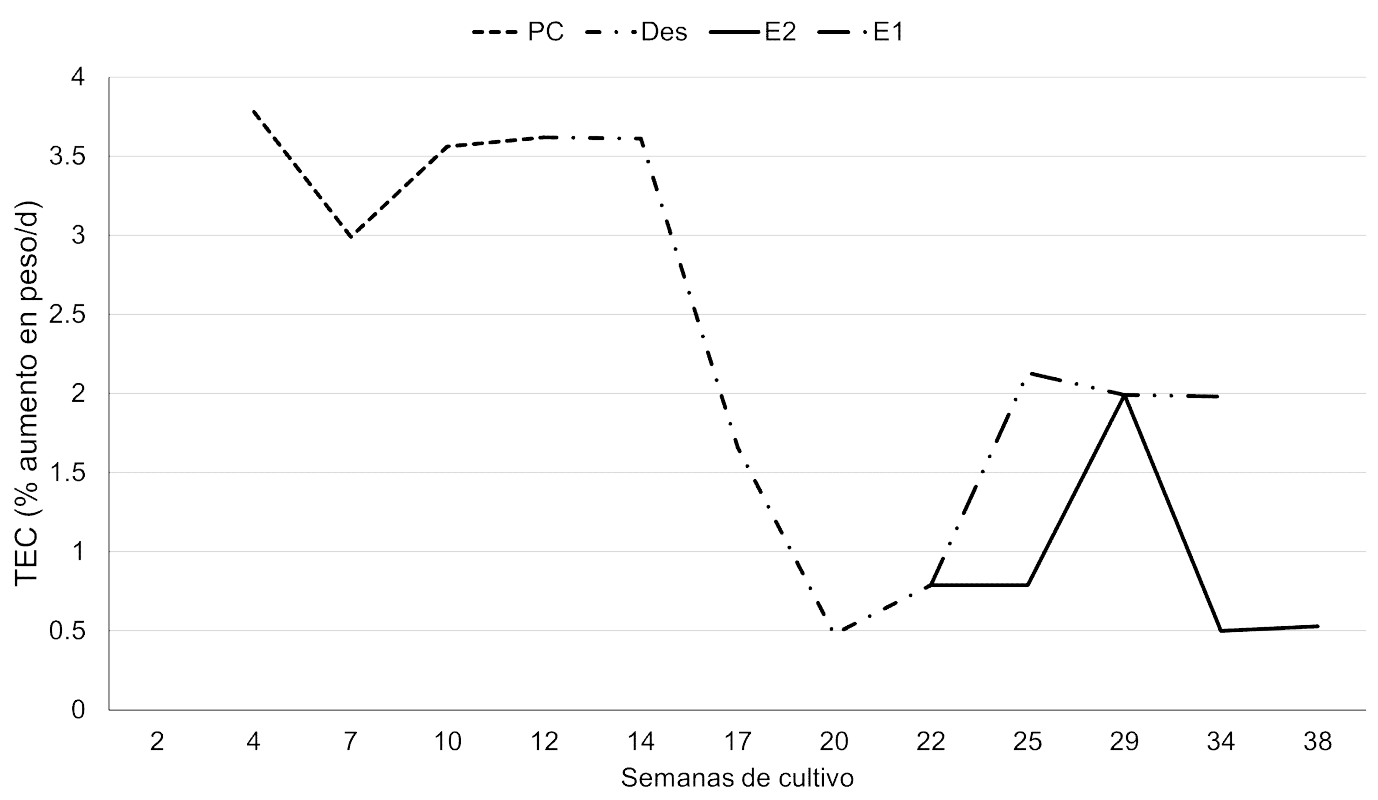

Figura 4. Tasa específica de crecimiento (\% aumento en peso/d) en las fases de precría, desarrollo y engorde de langostinos Macrobrachium rosenbergii (Costa Rica). (PC: precría; Des: fase de desarrollo; E1: fase de engorde para langostinos de talla grande; E2: fase de engorde para langostinos de talla pequeña)

mer año se pueden hacer 4 ciclos de precría, 3 de desarrollo y 1 de engorde (A), pero a partir del segundo año se pueden hacer 3.5 ciclos de engorde (B, C, D y $1 / 2 \mathrm{~F})$. Esto significa que la productividad se incrementa de un promedio de $112.8 \mathrm{~g} / \mathrm{m}^{2} /$ año a $\left(77.6 \mathrm{~g} / \mathrm{m}^{2}\right.$ en 3.5 ciclos/año) $271.6 \mathrm{~g} / \mathrm{m}^{2} / \mathrm{año}(2716 \mathrm{~kg} /$ ha/año) a partir del segundo año de cultivo y así sucesivamente.

\section{Crecimiento}

El aumento en el peso (Figura 2) y longitud (Figura 3) medidos semanalmente en todas las fases de estudio fue leve cuando se pasaron de alta densidad de siembra $\left(91.7 / \mathrm{m}^{2}\right)$ en precría a una densidad mediana $\left(12.5 / \mathrm{m}^{2}\right)$ en la fase de desarrollo, a pesar de darse una reducción brusca de $79.2 / \mathrm{m}^{2}$ en la densidad. El efecto más notorio en el crecimiento se dio cuando se separaron por tamaños para pasarlos a la fase de engorde con una reduc- ción poco significativa en la densidad de siembra (de $12.5 / \mathrm{m}^{2}$ a 6.1 y $4.8 / \mathrm{m}^{2}$ para los grandes y pequeños, respectivamente). No obstante, las diferencias no se notan claramente por la gran disparidad de pesos y tallas en las poblaciones muestreadas en la fase de engorde como lo indican las respectivas desviaciones estándar.

En ambas figuras se aprecia un aumento considerable en el crecimiento de los langostinos grandes sembrados aparte durante las primeras siete semanas, pero deteniéndose en la última, mientras que los langostinos pequeños mostraron una caída en la primera semana, logrando recuperarse después, con un crecimiento continuo hasta la cosecha. $\mathrm{Al}$ comparar las curvas de crecimiento con la curva teórica de crecimiento de $0.6 \mathrm{~g} / \mathrm{sema-}$ na sembrando de manera directa en similares densidades (Valverde y Varela, 2020), se puede observar que las medidas en todas las 


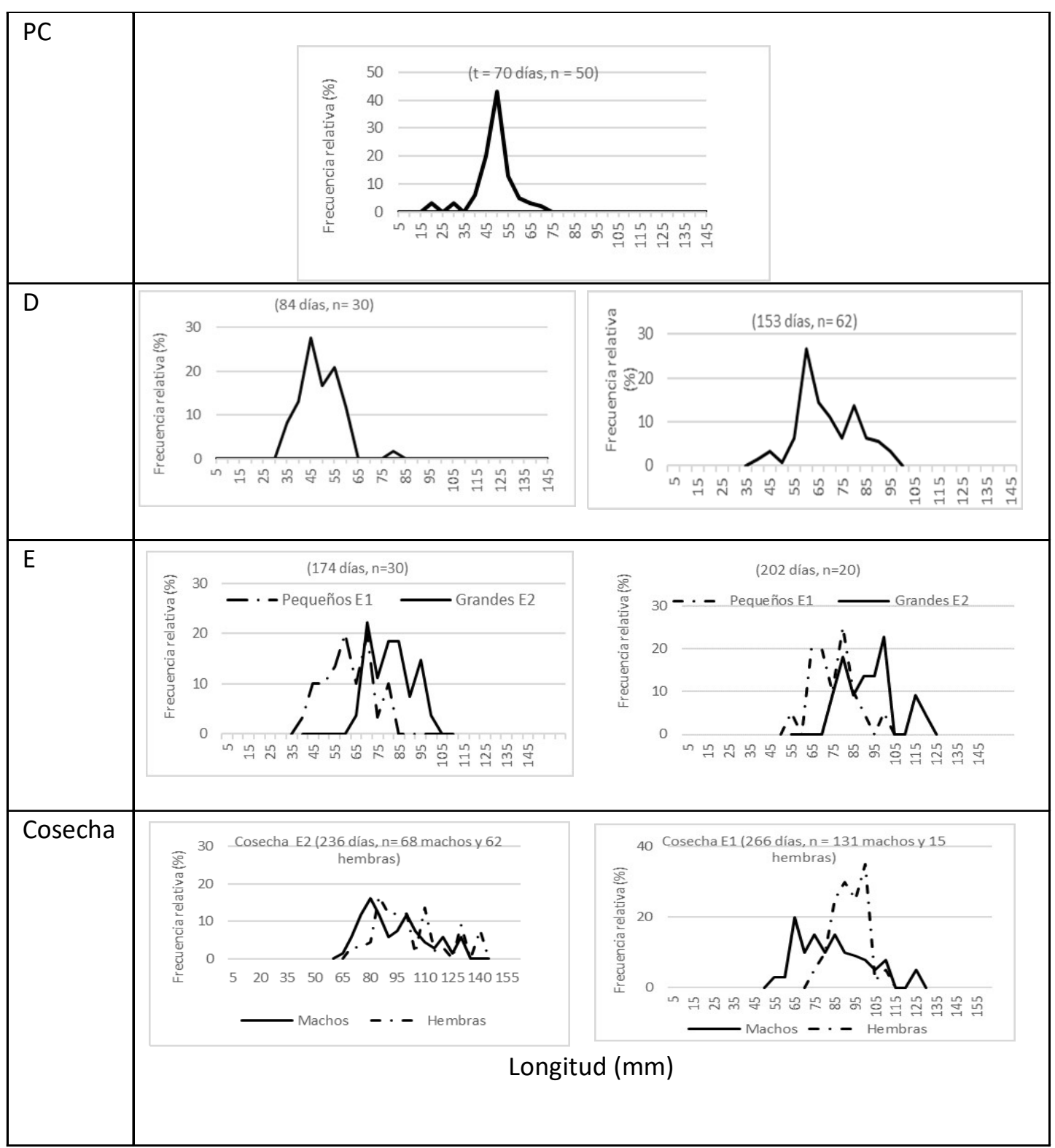

Figura 5. Distribución de la frecuencia relativa de longitud para el langostino de agua dulce Macrobrachium rosenbergii cultivado en fases y con separación de tamaños en la fase de engorde (PC: precría; D: fase de desarrollo; E: fase de engorde para langostinos de talla grande; E2: fase de engorde para langostinos de talla pequeña) 


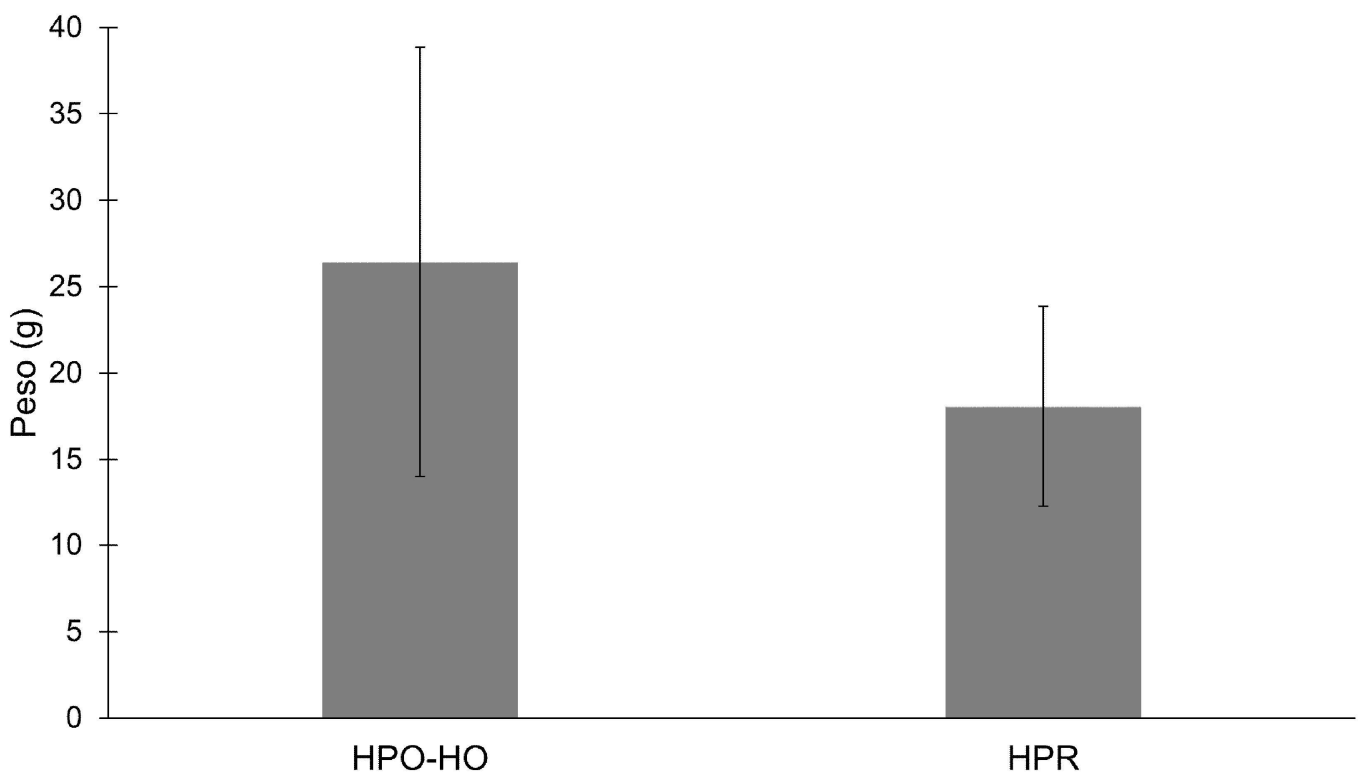

Figura 6. Peso de langostinos Macrobrachium rosenbergii hembras según estado reproductivo en el grupo de tallas grandes (fase de engorde) (HPO: post ovígeras, HO: ovígeras, HPR: pre ovígeras)

fases fueron inferiores con una diferencia final de $4.8 \mathrm{~g}$ en precría, $5.9 \mathrm{~g}$ en desarrollo y $8.3 \mathrm{~g}$ en el engorde de los pequeños, siendo solo superada por los langostinos grandes, aunque igualada cuando detuvieron el crecimiento en la última semana.

La TEC, medida como el porcentaje del aumento diario del peso, se muestra en la Figura 4. Se nota una disminución considerable en la TEC desde la precría (3.6\%g/día) al desarrollo $(0.8 \% \mathrm{~g} /$ día $)$ a pesar de la reducción significativa en la densidad de siembra. No se notó que el traslado de una alta densidad como en precría a una intermedia en desarrollo haya causado una compensación en el crecimiento de los langostinos. La TEC aumentó después de que se hizo la separación por tamaños antes de sembrarlos en engorde. Este aumento (a $2.0 \% \mathrm{~g} /$ día) fue muy notorio en las primeras tres semanas en E2 con los langostinos pequeños y de la tercera a la sétima semana en E1 donde se sembra- ron los langostinos grandes. Después se mantuvo en E2, pero decayó abruptamente en E1 a $0.5 \% \mathrm{~g} /$ día. Sin embargo, debido a la disparidad de tallas y pesos mostradas anteriormente en la fase de engorde, la TEC no permite apreciar con facilidad un posible crecimiento compensatorio cuando se separaron por tamaños para sembrar en esta fase.

\section{Dimorfismo}

En la Figura 5 se presenta la frecuencia relativa (\%) de la distribución de tallas ( $\mathrm{mm}$ ) para la población mixta durante los muestreos y para machos y hembras en la cosecha final. Puede observarse una distribución unimodal en PC y una bimodal al final del desarrollo, compuesta por grandes y pequeños. Esto significa que la separación por tamaños era difícil en la primera fase, mientras que se facilitó en la segunda poniendo la malla por donde pasaran los pequeños y se retuvieran los grandes. En los langostinos sepa- 


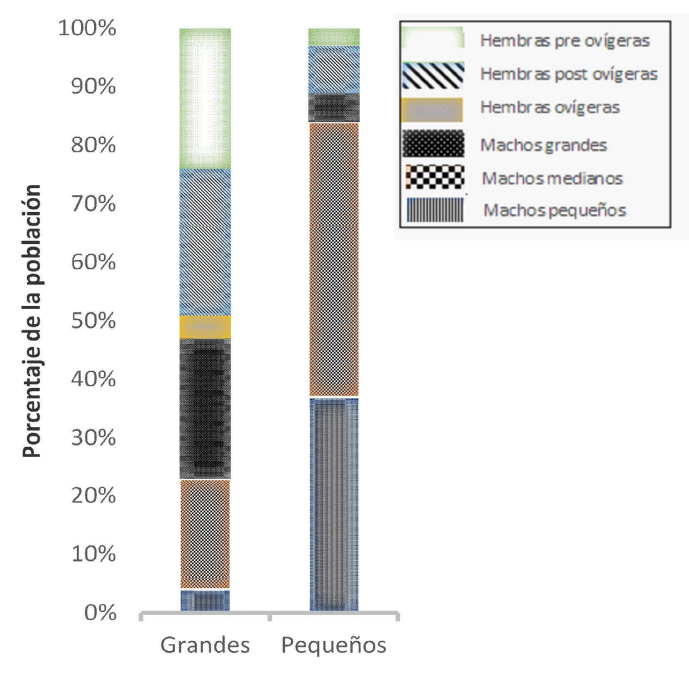

Figura 7. Porcentaje relativo de langostinos Macrobrachium rosenbergii machos y hembras según sus morfo tipos y estados reproductivos a la cosecha en los grupos separados como grandes y pequeños

rados por talla en la fase de engorde se nota la superioridad de los grandes mostrando el grupo de los MG sobre los pequeños. En la cosecha de los grandes no se observó la distribución unimodal característica de las hembras como se aprecia en el de los pequeños, sino que estuvo conformada por grupos de estados reproductivos y tamaños distintos, como se muestra en la Figura 6.

En la Figura 7 se presenta el porcentaje relativo de machos y hembras según sus morfo tipos y estados reproductivos a la cosecha en los grupos que fueron separados como grandes y pequeños antes de sembrarlos en la fase de engorde. Se nota que la mayor proporción $(90 \%)$ del grupo de los pequeños fueron machos y que el porcentaje de MP y MM (37 y 47\%, respectivamente) se incrementó considerablemente en este grupo con respecto al de los grandes (4 y 19\%, respectivamente). Así mismo, disminuyó la proporción de MG (de 24 a 6\%). Las hembras también mostraron una reducción considerable de 53 a $10 \%$ en el grupo de los pequeños sin encontrarse hembras ovígeras en este grupo.

El peso y la longitud promedio de la población de machos por grupos de grandes y pequeños se muestran en la Figura 8. Los valores son similares, excepto por los MM que muestran una leve superioridad en el grupo de los pequeños con respecto a los grandes. En el Cuadro 5 se muestra las mediciones de las características de la producción a la cosecha durante el procesamiento de los langostinos en los grupos grandes y pequeños.
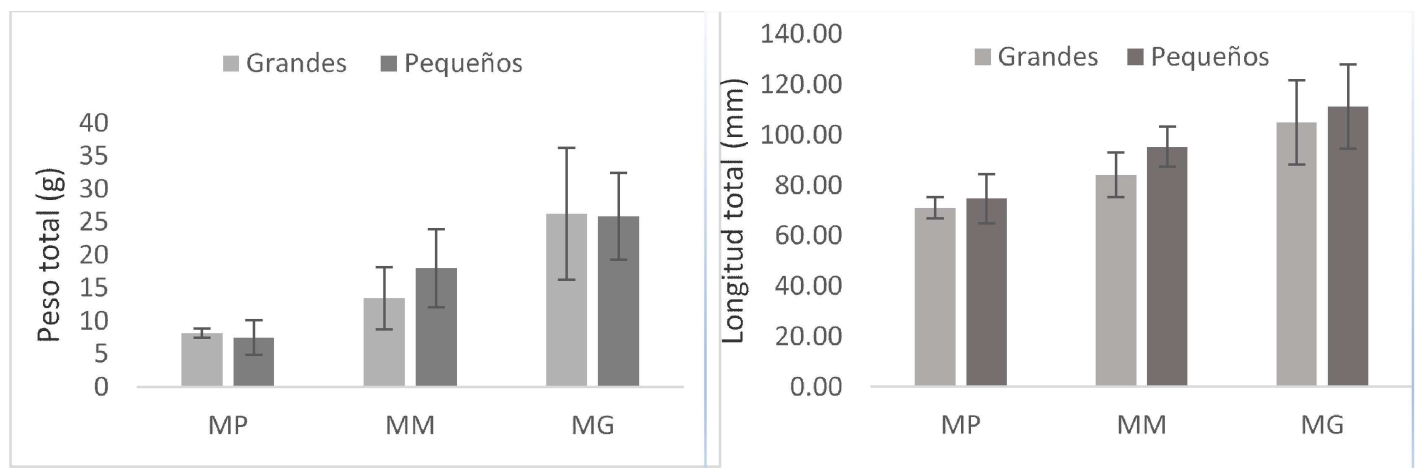

Figura 8. Peso y longitud de los morfo tipos de langostinos Macrobrachium rosenbergii machos en los grupos de grandes y pequeños 
Cuadro 5. Características de la producción de los langostinos Macrobrachium rosenbergii durante el procesamiento de los grupos grandes y pequeños.

\begin{tabular}{|c|c|c|c|c|c|c|c|c|c|c|}
\hline \multirow{2}{*}{ Sexo } & \multirow{2}{*}{ Lote } & \multirow{2}{*}{$\mathrm{n}$} & \multirow{2}{*}{$\begin{array}{l}\text { Morfo- } \\
\text { tipo }\end{array}$} & \multicolumn{4}{|c|}{ Peso (g) } & \multirow{2}{*}{$\begin{array}{c}\text { Cola } \\
(\%)\end{array}$} & \multicolumn{2}{|c|}{ Longitud (mm) } \\
\hline & & & & Total & Cabeza & Tenazas & Abdomen & & Total & Tenazas \\
\hline \multirow{4}{*}{ 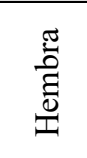 } & \multirow{2}{*}{ Grande } & \multirow{2}{*}{70} & \multirow{2}{*}{ Todas } & $21.45 \pm$ & $7.75 \pm$ & $0.53 \pm$ & $7.72 \pm$ & \multirow{2}{*}{26.0} & $100.88 \pm$ & $78.70 \pm$ \\
\hline & & & & 9.95 & 2.30 & 0.27 & 2.21 & & 1.83 & 1.66 \\
\hline & \multirow{2}{*}{ Pequeño } & \multirow{2}{*}{15} & \multirow{2}{*}{ Todas } & $16.57 \pm$ & $7.52 \pm$ & $0.43 \pm$ & $10.33 \pm$ & \multirow{2}{*}{42.1} & $94.00 \pm$ & $70.77 \pm$ \\
\hline & & & & 2.24 & 1.09 & 0.17 & 4.68 & & 0.39 & 0.76 \\
\hline \multirow{11}{*}{$\begin{array}{l}0 \\
\frac{0}{0} \\
\stackrel{\pi}{\Sigma}\end{array}$} & \multirow{5}{*}{ Grande } & 34 & MG & $\begin{array}{c}26.26 \pm \\
9.97\end{array}$ & $\begin{array}{c}11.21 \pm \\
4.36\end{array}$ & $\begin{array}{c}1.07 \pm \\
0.70\end{array}$ & $\begin{array}{c}10.19 \pm \\
3.23\end{array}$ & 26.4 & $\begin{array}{c}104.91 \pm \\
1.68\end{array}$ & $\begin{array}{c}101.06 \pm \\
3.71\end{array}$ \\
\hline & & \multirow{2}{*}{27} & \multirow{2}{*}{ MM } & $13.44 \pm$ & $6.64 \pm$ & $0.56 \pm$ & $6.45 \pm$ & \multirow{2}{*}{31.3} & $84.07 \pm$ & $66.37 \pm$ \\
\hline & & & & 4.71 & 2.41 & 0.49 & 2.29 & & 0.89 & 1.13 \\
\hline & & \multirow{2}{*}{5} & \multirow{2}{*}{ MP } & $8.18 \pm$ & $3.54 \pm$ & $0.13 \pm$ & $4.56 \pm$ & \multirow{2}{*}{38.5} & $71.00 \pm$ & $53.00 \pm$ \\
\hline & & & & 0.72 & 0.82 & 0.06 & 0.81 & & 0.42 & 0.67 \\
\hline & \multirow{6}{*}{ Pequeño } & \multirow[t]{2}{*}{8} & \multirow[t]{2}{*}{ MG } & $27.89 \pm$ & $13.42 \pm$ & $2.75 \pm$ & $15.76 \pm$ & \multirow[t]{2}{*}{35.8} & $111.25 \pm$ & $124.37 \pm$ \\
\hline & & & & 10.61 & 5.75 & 2.77 & 16.6 & & 1.66 & 4.49 \\
\hline & & \multirow[t]{2}{*}{68} & \multirow[t]{2}{*}{ MM } & $18.01 \pm$ & $7.02 \pm$ & $0.45 \pm$ & $7.18 \pm$ & 28.2 & $95.22 \pm$ & $76.23 \pm$ \\
\hline & & & & 5.94 & 1.36 & 0.22 & 1.35 & & 0.80 & 1.47 \\
\hline & & 53 & MP & $7.50 \pm$ & $3.44 \pm$ & $0.20 \pm$ & $4.02 \pm$ & 36.1 & $74.68 \pm$ & $51.74 \pm$ \\
\hline & & & & 2.63 & 1.31 & 0.12 & 1.31 & & 0.98 & 1.08 \\
\hline
\end{tabular}

La proporción relativa de la longitud de las tenazas con respecto a la del cuerpo como promedio en los dos grupos fue de $0.77 \pm$ 0.02 las hembras, $1.04 \pm 0.11$ los $\mathrm{MG}, 0.80 \pm$ 0.01 los MM y $0.72 \pm 0.04$ los MP. De igual manera, el porcentaje del peso de las tenazas con respecto al del cuerpo fue de $2.5 \pm 0.1$ las hembras, $7.0 \pm 4.1$ los MG, $3.3 \pm 1.2$ los MM y $2.1 \pm 0.8$ los MP. Con respecto al porcentaje de cola en orden descendente, este fue de 54.7 los MP, 49.2 las hembras, 47.7 los MG y 43.9 los MM. El promedio del rendimiento en cola para todos los langostinos fue de $48.9 \%$. El peso promedio de la cabeza y las tenazas para las hembras fue de $8.1 \mathrm{~g}$ y para los machos de $14.2 \mathrm{~g}$ los MG, $7.3 \mathrm{~g}$ los MM y $3.7 \mathrm{~g}$ los MP.

\section{Discusión}

Las principales variables de la calidad del agua medidas en la pila de precría y los estanques de desarrollo y engorde forrados con plástico no mostraron mayores diferencias con aquellas reportadas en estudios anteriores (Valverde y Varela, 2020) en esta región del país. Los valores de la temperatura del agua estuvieron dentro del rango considerado óptimo (entre 26 y $32{ }^{\circ} \mathrm{C}$ ) en las mañanas para el metabolismo de los langostinos (Boyd y Zimmermann, 2000) y dentro del cual $\left(28-30{ }^{\circ} \mathrm{C}\right)$ se da la mayor distribución de tallas (Alston y Sampaio, 2000).

La turbidez del agua $(34-45 \mathrm{~cm})$ estuvo dentro del rango $(26-50 \mathrm{~cm})$ recomendado (Samad et al., 2016). No obstante, en el mes de octubre los niveles de oxígeno disuelto bajaron a $1.8 \mathrm{mg} / 1$ cuando la turbidez fue de $34 \mathrm{~cm}$ y provocaron mortalidad en un momento de mudas fuertes. Este podría ser un indicativo de que es preferible mantener el agua relativamente clara $(>40 \mathrm{~cm})$ para el cultivo de langostinos. Estudios han demostrado que valores menores de $2.0 \mathrm{mg} / \mathrm{l}$ en las mañanas causan estrés en los langostinos cuando están duros y mortalidad cuando están suaves debido a periodos de fuertes mu- 
das (Boyd y Zimmermann, 2000). El bajo nivel de OD es el principal factor que causa mortalidad en los langostinos y se debe tanto a la abundancia excesiva de fitoplancton como a la acumulación de sedimento durante el proceso de descomposición (Valenti y New, 2000). Se recomienda que las concentraciones de OD siempre estén arriba de $3 \mathrm{mg} / \mathrm{l}$ para no afectar los rendimientos de producción (Al-Mohsen, 2009).

La menor sobrevivencia (61\%) en el estanque de engorde con langostinos grandes con respecto al de langostinos pequeños $(100 \%)$ fue producto de la mortalidad observada en un día de cultivo. Las mortalidades relacionadas con enfermedades son poco frecuentes en langostinos debido sobre todo a las bajas densidades de siembra utilizadas. Una de las enfermedades más comunes son las manchas negras causadas frecuentemente por bacterias que atacan la cutícula (Johnson y Bueno, 2000). En Bangladesh, la enfermedad de la mancha negra o de la branquia negra es la más común desde que siembran las PL's hasta la cosecha, causando mortalidades masivas y las mayores pérdidas económicas (Akter et al., 2014).

La medida de control utilizada en este estudio como tratamiento profiláctico fue la aplicación semanal de cal hidratada $(\mathrm{Ca} \mathrm{OH})$. Las aplicaciones rutinarias de cal al agua de los estanques ayudan a aumentar la concentración del calcio disuelto, aceleran la descomposición de la materia orgánica en el fondo y eliminan a los patógenos cuando produce un cambio brusco en el pH $(>10)$. En este estudio se hizo una aplicación semanal de $40 \mathrm{~g}$ de cal al estanque $\left(0.55 \mathrm{~g} / \mathrm{m}^{2}\right)$. La misma está por debajo del tratamiento recomendado de $100-150 \mathrm{~g} / \mathrm{m}^{2}$ (Boyd y Zimmermann, 2000). Un tratamiento como $75 \mathrm{~g} / \mathrm{m}^{2}$ aumentaría el $\mathrm{pH}$ del estanque en más de 10 debido a la reacción de la cal con el agua y esta es la razón por la que este material se recomienda como desinfectante del suelo o agua desempeñando un papel crucial en el manejo de estanques acuícolas (Boyd, 2017).
Las densidades de siembra elegidas en el engorde $\left(4.8\right.$ y $\left.6.1 / \mathrm{m}^{2}\right)$ están dentro del rango utilizado comúnmente con fines comerciales (Valenti y New, 2000). Estudios han mostrado la reducción de la rentabilidad con el aumento en la densidad de siembra arriba de $5 / \mathrm{m}^{2}$ (Rhodes, 2000) y nula rentabilidad a $6 / \mathrm{m}^{2}$ sembrando de manera directa (Valverde y Varela, 2020). El cultivo en fases con la separación por tallas puede hacer más positiva la rentabilidad en relativamente altas densidades de siembra aumentando la intensidad del cultivo (Karplus et al., 2000).

Los pesos finales obtenidos de las fases de precría y desarrollo en este estudio se pueden comparar con los reportados a nivel comercial por la empresa Sabana Grande en Puerto Rico en la década de los noventa (Alston y Sampaio, 2000). En la precría se obtuvo un peso promedio de $2.5 \mathrm{~g}$ sembrando juveniles I provenientes de maternidad con un peso promedio de $0.1 \mathrm{~g}$ a una densidad de $91.7 / \mathrm{m}^{2}\left(200 / \mathrm{m}^{2}\right.$ en Sabana Grande) al cabo de 10 semanas. El peso inicial es el comúnmente utilizado en zonas tropicales (Samad et al., 2016), aunque en zonas templadas por lo general usan un peso mayor (0.3-0.4 g) (Tidwell et al., 2015). Un peso final similar (2.3 g) fue obtenido sembrando PL's a 50/ $\mathrm{m}^{2}$ en jaulas durante 8 semanas (Marques y Lombardi, 2011).

El peso promedio final de la fase de desarrollo fue de $7.3 \mathrm{~g}$ ( 9 a $15 \mathrm{~g}$ en Sabana Grande) en 12 semanas sembrando juveniles II ( $2.5 \mathrm{~g})$ provenientes de precría a una densidad de siembra de $12.5 / \mathrm{m}^{2}\left(20-30 / \mathrm{m}^{2}\right.$ en Sabana Grande). Obviando esta fase de desarrollo, Marques y Lombardi (2011) reportaron un peso promedio final de $18.5 \mathrm{~g}$ al cabo de 34 semanas sembrando los juveniles provenientes de precría con similares tamaños ( $2.3 \mathrm{~g}$ ) y densidades de siembra de $12 / \mathrm{m}^{2}$.

A diferencia de Sabana Grande donde se hacían cosechas parciales de los juveniles grandes en desarrollo para pasarlos a engorde, en este estudio se hizo cosecha total para 
separar grandes de pequeños y sembrarlos por separado bajando la densidad de siembra para llevarlos a talla comercial. Se obtuvo pesos finales de $21.9 \mathrm{~g}$ sembrando los grandes $(10.4 \mathrm{~g})$ a una densidad de $6.1 / \mathrm{m}^{2}$ al cabo de 12 semanas y $15.3 \mathrm{~g}$ sembrando los pequeños $(4.1 \mathrm{~g})$ a $4.8 / \mathrm{m}^{2}$ durante 16 semanas. El peso final del grupo de los grandes fue levemente superior al obtenido con siembra directa a $6.0 / \mathrm{m}^{2}(20.1 \mathrm{~g}$; Valverde y Varela, 2020). Los valores promedio del engorde fueron de $18.6 \mathrm{~g}$ sembrando a $5.5 / \mathrm{m}^{2}$ con un peso inicial de $7.3 \mathrm{~g}$ en un periodo de $14 \mathrm{se}-$ manas. Un peso similar ( $18.5 \mathrm{~g})$ fue obtenido pasando directamente de precría $(2.3 \mathrm{~g})$ a engorde a $12 / \mathrm{m}^{2}$ en 34 semanas (Marques y Lombardi, 2011), pero el periodo de cultivo fue de ocho semanas adicionales en comparación con el del presente estudio de 26 semanas (12 en desarrollo y 14 en engorde). Pesos finales cercanos a los $18 \mathrm{~g}$ han sido obtenidos en la mayoría de los países latinoamericanos sembrando de 6 a $12 / \mathrm{m}^{2}$ al cabo de 20-35 semanas de cultivo (Jia et al., 1988).

El valor considerablemente más alto de la TCR en precría (24.4) que en las otras dos fases (1.9) indica la inexistencia de crecimiento compensatorio con la reducción en las densidades de siembra. Por lo general, la TRC ha mostrado una relación inversamente proporcional con el tamaño inicial de los langostinos sembrados (Marques y Lombardi, 2011) que con las densidades de siembra. Cuando los langostinos se separaron por tamaños en la siembre para engorde, la población de los pequeños mostró una TRC doblemente superior (2.7) que los sembrados con tallas más grandes (1.1). Desde antes se sugería la ocurrencia de crecimiento compensatorio cuando los langostinos pequeños se separaban de los grandes y se sembraban a densidades más bajas (Malecha et al., 1989).

Los valores de sobrevivencia (81-88\%) encontrados en todas las fases son similares a los reportados por Valverde y Varela (2020) en la baja $(89 \%)$ y alta $(80 \%)$ densidad de siembra directa utilizadas. Sobrevivencias entre 60 y $75 \%$ son comunes en los cultivos comerciales en Bangladesh dentro de un amplio rango que puede ir de 55 a $90 \%$, siendo mayor en las menores densidades de siembra (Samad et al., 2016). Las sobrevivencias han oscilado entre 69 y $84 \%$ en varios lotes de otros países asiáticos (Vietnam, Indonesia, Malasia y Fiji) al cabo de 20 semanas sembrando a $5 / \mathrm{m}^{2}$ (Singh, 2011). En regiones templadas, las sobrevivencias (86-95\%) de los lotes comerciales han superado a los silvestres $(77-80 \%)$ como una muestra de la domesticación a las condiciones de cultivo (Tidwell et al., 2014).

Las conversiones alimenticias (de 08 a 1.5:1.0) fueron menores a las reportadas por Valverde y Varela (2020) con siembra directa (de 1.6 a 2.2:1.0) utilizando tasas de alimentación más bajas (de 0.4 a $2.0 \mathrm{~g} / \mathrm{m}^{2} / \mathrm{d}$ ) dado que en siembra directa fueron considerablemente más altas (de 15.1 a $25.9 \mathrm{~g} / \mathrm{m}^{2} / \mathrm{d}$ ). En sistemas de biofloc, las conversiones alimenticias en precría fueron de 1.3:1.0 sembrando a $50 / \mathrm{m}^{2}$ (Negrini et al., 2017). Conversiones en rangos de 1.8 a 2.3:1.0 han sido reportadas en otros estudios con similares densidades de siembra (Ahmed, 2013; Nair y Salin, 2012). Conversiones alimenticias elevadas ( $>2.0: 1.0)$ no reflejan necesariamente el consumo de alimento porque gran parte queda remanente, se descompone y contribuye a aumentar la productividad natural satisfaciendo los requerimientos nutricionales para el crecimiento de los langostinos (D'Abramo y New, 2000).

Las aplicaciones de alimento utilizando la tabla de alimentación indicada fueron bajas. En general, la cadena alimenticia que se genera en el detritus constituye la fuente principal de alimento para los langostinos. El alimento suministrado usualmente no lo ingieren hasta que se descompone y ha sido incorporado en el detritus. Por lo tanto, el manejo de los estanques para langostinos se debe enfocar fundamentalmente a la comunidad bentónica y a la cadena alimenticia del detritus como fuente de nutrientes esenciales (Valenti y New, 2000). En sistemas semi-intensivos de cultivo con densidades de siem- 
bra arriba de $4 / \mathrm{m}^{2}$ y producciones de 500 a $5000 \mathrm{~kg} / \mathrm{ha} / \mathrm{año}$ se recomienda combinar la aplicación de alimentos formulados con la de fertilizantes (Singh, 2011).

La producción en precría fue superior a la obtenida usando otros sistemas de cultivo como jaulas $\left(52.5 \mathrm{~g} / \mathrm{m}^{2}\right.$ con peso final de $2.3 \mathrm{~g}$ y $96 \%$ de sobrevivencia) (Marques y Lombardi, 2011) y biofloc en tanques $\left(13.1 \mathrm{~g} / \mathrm{m}^{2}\right.$ de $2.2 \mathrm{~g}$ con $73 \%$ de sobrevivencia) (Negrini et al., 2017) sembrando en densidades más bajas $\left(50 / \mathrm{m}^{2}\right)$ durante menores tiempos de cultivo (8 semanas). Estudios en países asiáticos han demostrado que la fase de precría es beneficiosa para mejorar los cultivos comerciales y su rentabilidad en regiones tropicales (Madhusoodana y Ranjeet, 2005). Por lo tanto, la incorporación de las precrías en las fincas de producción comercial de langostinos debe ser una parte integral del sistema de cultivo por su viabilidad técnica y económica.

Sobre la producción obtenida de la fase de desarrollo existen pocos estudios de comparación dado que no ha sido una etapa muy utilizada. Quizás la principal ventaja de la fase de desarrollo es la facilidad con que se pueden separar langostinos pequeños de grandes con una red en un tanque con agua por donde pasen los primeros y se retengan los segundos. Este proceso en otras circunstancias como cosechas selectivas ha resultado laborioso, periódico y poco efectivo (Shivakumar et al., 2012).

La inexistencia de crecimiento compensatorio de precría a desarrollo sin separación de tallas podría ser un indicativo de que esta fase intermedia es innecesaria. Mejores resultados se han obtenido pasando directamente de precría a engorde con una drástica disminución en la densidad de siembra (de $92 / \mathrm{m}^{2}$ a 4-6/ $\mathrm{m}^{2}$ ) como fue observado en el cultivo en fases en camarones marinos (Valverde y Alfaro, 2015). La separación a los 60 días en precría con un tamaño de 0.6-0.7 g dio un incremento significativo en los índices de producción, mientras que con un periodo adicional de 72 días y un tamaño de $3.1 \mathrm{~g}$ no se mejoraron los rendimientos, sino que se incrementó la conversión alimenticia. Este efecto negativo de sembrar animales considerablemente más grandes se atribuyó a la aparición de una maduración temprana que contribuyó en el estancamiento de la curva de crecimiento (Tidwell et al., 2005).

La producción en engorde con un promedio de $77.6 \pm 5.2 \mathrm{~g} / \mathrm{m}^{2}$ fue inferior a la obtenida a $6 / \mathrm{m}^{2}\left(96.1 \mathrm{~g} / \mathrm{m}^{2}\right)$ (Valverde y Varela, 2020). Estas producciones son similares a las establecidas por Marques y Moraes-Valenti (2012) en Brasil, quienes con densidades de siembra de 5 a $10 / \mathrm{m}^{2}$ lograron producciones de $80-150 \mathrm{~g} / \mathrm{m}^{2}$. En zonas templadas con ciclos de producción de 112 a 120 140 días se han obtenido producciones de 47.5 a $100 \mathrm{~g} / \mathrm{m}^{2}$ sembrando a $2.5 / \mathrm{m}^{2}$ (Tidwell y D'Abramo, 2010; Tidwell et al., 2014).

Los rendimientos de la producción fueron de $2.1 \mathrm{~kg}$ en precría, $6.5 \mathrm{~kg}$ en desarrollo y $14.2 \mathrm{~kg}$ por cada millar de animales sembrados. El rendimiento en engorde fue inferior al obtenido $(16.0 \mathrm{~kg} /$ millar) por Valverde y Varela (2020) en una similar densidad de siembra $\left(6.0 / \mathrm{m}^{2}\right)$. Con respecto a los separados por tamaños en engorde, los índices indican una biomasa total relativa nueve veces mayor en los langostinos pequeños (2.7) que en los grandes (0.3) y una similitud en el rendimiento por cada 1000 langostinos sembrados $(52 \%$ o $7.4 \mathrm{~kg}$ en la fracción de los grandes y $48 \%$ o $6.8 \mathrm{~kg}$ en la de los pequeños).

La productividad del cultivo en fases fue de $2716 \mathrm{~kg} / \mathrm{ha} /$ año a partir del segundo año de cultivo haciendo 3.5 ciclos al año. Este valor supera al obtenido con siembra directa la cual fue estimada en $2595 \mathrm{~kg} / \mathrm{ha} / \mathrm{año}$ a $6.0 / \mathrm{m}^{2}$ (Valverde y Varela, 2020). En zonas tropicales con similares densidades de siembra y condiciones de clima, las productividades han fluctuado entre 1000 y $3000 \mathrm{~kg} / \mathrm{ha} /$ año con sistemas semi-intensivos de cultivo (Valenti y New, 2000). En zonas templadas se reportan productividades de 1500 a 4500 $\mathrm{kg} / \mathrm{ha} /$ año sembrando a $2.5 / \mathrm{m}^{2}$ con $2-3$ ciclos al año utilizando precrías (Tidwell, 2012). 
Sembrando en densidades superiores a 10/ $\mathrm{m}^{2}$ no se logró incrementar la productividad ya que los valores fueron inferiores a los 4600 $\mathrm{kg} / \mathrm{ha} / \mathrm{año}$ obtenidos con esta densidad (Samad et al., 2016).

La productividad obtenida mediante el cultivo en fases superó el nivel de producción en equilibrio $\left(2321 \mathrm{~kg} / \mathrm{ha} /\right.$ año a $\left.6.0 / \mathrm{m}^{2}\right)$ que fue establecido sembrando de manera directa (Valverde y Varela, 2020). Esto indica que se logró el mejoramiento en las producciones con densidades de siembra más altas instalando en las unidades productivas las tres fases de cultivo. En el caso de los langostinos pequeños se logró llevar el tamaño de 4.1 a $15.3 \mathrm{~g}$ e incrementar la producción a $103.7 \mathrm{~g} / \mathrm{m}^{2} /$ año $(1037 \mathrm{~kg} / \mathrm{ha} /$ año $)$ dentro de valores donde existe suficiente mercado. De otra forma se hubieran tenido que descartar como fue recomendado por Malecha (Malecha, 1988), quien indicaba que era más rentable descartar los langostinos pequeños y usar solo los más grandes para sembrar los estanques de engorde. El efecto de separarlos por tamaños sobre la producción y la rentabilidad del cultivo no había sido investigado en los países tropicales en aquel tiempo.

El crecimiento semanal mostró la relación inversa con respecto a la densidad de siembra ya que fue de $0.25 \pm 0.02 \mathrm{~g} /$ semana en precría a $91.7 / \mathrm{m}^{2}, 0.62 \pm 0.01 \mathrm{~g} / \mathrm{semana}$ en desarrollo a $12.5 / \mathrm{m}^{2}$ y $1.40 \pm 0.64 \mathrm{~g} / \mathrm{se}-$ mana a $5.5 / \mathrm{m}^{2}$ como promedio en engorde. Los grandes crecieron prácticamente el doble $(1.85 \mathrm{~g} / \mathrm{semana})$ que los pequeños $(0.95$ $\mathrm{g} /$ semana). No obstante, las diferencias en la tasa de crecimiento no se notan claramente por la gran disparidad de pesos y tallas en las poblaciones muestreadas en la fase de engorde. Las curvas de crecimiento muestran que los langostinos grandes detuvieron su crecimiento la última semana, mientras que los pequeños tuvieron una tendencia creciente que probablemente hubiera reducido las diferencias de tamaño si no se no se hubiera interrumpido su fase de crecimiento con la cosecha final.
En comparación con la curva teórica de crecimiento de $0.6 \mathrm{~g} / \mathrm{semana}$ que se puede considerar como el grupo control compuesto por una población mixta sembrada de manera directa en similares densidades y bajo condiciones adecuadas de cultivo (Valverde y Varela, 2020), las medidas en todas las fases fueron inferiores con una diferencia final de $4.8 \mathrm{~g}$ en precría, $5.9 \mathrm{~g}$ en desarrollo y $8.3 \mathrm{~g}$ en el engorde de los pequeños, siendo solo superada por los langostinos grandes e igualada cuando detuvieron el crecimiento en la última semana. El estancamiento del crecimiento pudo deberse a que alcanzaron la madurez sexual y se dedicaron a la reproducción bajando su incidencia en el peso final a cosecha (Tidwell et al., 2005). Tomando en cuenta estas curvas de crecimiento y obviando la disparidad de tallas, podría considerarse que existió crecimiento compensatorio completo en el grupo de los langostinos grandes cuando se les quitó la restricción de la mayor densidad de siembra y la presencia de los pequeños. En menos tiempo (12 semanas) superaron y luego igualaron al grupo considerado como control (Prates et al., 2020). No hubo compensación del crecimiento en el grupo de los pequeños a pesar de que en 16 semanas mostraban una curva de crecimiento en ascenso y probablemente requerían de más tiempo para alcanzar un peso más cercano al de la población control. Estudios han indicado que extiendo el periodo de cultivo de 9 a 19 semanas en precría se incrementó el peso de 0.7 a $3.1 \mathrm{~g}$ (Tidwell et al., 2003).

De acuerdo con los valores de la TEC, en este estudio no se encontró crecimiento compensatorio al pasar los langostinos de precría a desarrollo y engorde con las respectivas reducciones en las densidades de siembra. El valor más alto de la TEC en la PC $(3.6 \% \mathrm{~g} /$ día) es atribuible a la gran diferencia de tamaño entre los juveniles I $(0.1 \mathrm{~g})$ sembrados y los juveniles II ( $2.5 \mathrm{~g})$ cosechados al cabo de 70 días de cultivo. Este valor de la TEC es ligeramente superior al $3.3 \% \mathrm{~g} / \mathrm{d}$ obtenido en precría sembrando a $50 / \mathrm{m}^{2}$ en biofloc al cabo de 60 días (Negrini et al., 
2017) y al reportado de $3.2-3.4 \% \mathrm{~g} /$ día en la fase de engorde con siembra directa en Bangladesh usando densidades de 10 a $14 / \mathrm{m}^{2}$ al cabo de 6 meses de cultivo (Samad et al., 2016).

Valores de la TEC más bajos del 2\%g/d como los obtenidos en las fases de desarrollo y engorde en este estudio no son comunes en países asiáticos tropicales donde los pesos de cosecha pueden fluctuar de 50 a $60 \mathrm{~g}$ en altas $\left(12 / \mathrm{m}^{2}\right)$ densidades de siembra (Samad et al., 2016). Estos valores bajos podrían atribuirse a un deterioro genético por endogamia al venirse utilizando el mismo material de las pequeñas cantidades de reproductores que fueron introducidas (New y Kutty, 2010). No obstante, se ha demostrado que la domesticación parece tener un impacto más determinante en los rendimientos de producción y la menor disparidad de tallas que el deterioro genético debido a que las especies se han adaptado mejor a las condiciones de cultivo (Tidwell et al., 2014). Se debería investigar el efecto de las contaminaciones en los viveros y en las hembras reproductoras que afectan las larvas (Varela y Valverde, 2018) como ha sido descrito en China con la infección de Enterobacter cloacae (Gao et al., 2020). Otra posibilidad es el pequeño tamaño de las hembras utilizadas como reproductoras basándose en su estado de madurez, sin tomar en cuenta el mayor tamaño ni que sean las primeras en crecer hasta alcanzar la madurez sexual (Singh, 2011).

La distribución frecuencia-longitud del langostino $M$. rosenbergii cultivado en la fase de precría mostró tamaños similares para la población de ambos sexos, pero a medida que los individuos fueron madurando en la fase de desarrollo se separaron por tamaños, quedándose rezagada una población compuesta sobre todo por los machos pequeños dominados (Ra'anan y Cohen, 1985). Al pasar a la fase de engorde, las hembras van reduciendo su crecimiento a medida que van madurando, quedan fecundadas y dedican la mayor parte de su energía al cuidado e incubación de los huevos. Los MG continúan creciendo hasta llegar a ser más grandes que las hembras y los otros machos. Se vuelven dominantes y esto reduce el crecimiento de los más pequeños cuando están juntos dando una gran variación de tallas entre los machos. Las hembras por lo general crecen muy parejo, pero el tiempo en alcanzar la maduración más rápido para quedar fecundadas puede hacer que las hembras ovígeras y pos-ovígeras lleguen a ser más grandes que las pre-ovígeras, las cuales están apenas iniciando la reproducción (Ra'anan y Cohen, 1985). Este comportamiento fue observado en el grupo de los langostinos grandes a la cosecha en este estudio; no obstante, la poca cantidad de hembras en el grupo de los pequeños no afectó su distribución unimodal.

Las amplias variaciones de tallas entre los machos, mostradas por las desviaciones estándar, no permitieron identificar claramente el crecimiento compensatorio cuando fueron separados por tallas. Las diferencias en la distribución de los morfo tipos de machos podrían dar una idea más clara del cambio compensatorio. Estudios han indicado que la posición relativa de los morfo tipos dentro de la población es más importante que el tamaño y el peso absolutos a la hora de establecer la productividad y el crecimiento compensatorio de los langostinos (Karplus et al., 1986). Diferencias en el peso promedio final a la cosecha, los rendimientos de producción y la rentabilidad del cultivo se han dado en las poblaciones separadas por tamaños a causa de los cambios en las distribuciones de los morfo tipos en la población (Daniels y D’Abramo, 1994).

Las proporciones encontradas por sexo fueron de $90 \%$ machos en el grupo de los pequeños con una relación 9.0:1.0 macho: hembra y $47 \%$ machos en el de los grandes con una relación 0.9:1.0 macho: hembra. En otros estudios, la proporción de hembras ha sido ligeramente superior a la de los machos al cabo de 105 días de cultivo separando por tallas en precría y sembrando a $2 / \mathrm{m}^{2}$ (Karplus et al., 1986). 
Con respecto a solo los machos, las proporciones fueron de $37 \% \mathrm{MP}, 47 \% \mathrm{MM}$ y 6\% MG (4:5:1) en el grupo de los pequeños, mientras que en el de los grandes fue lo contrario: 4\% MP, 19\% MM y 24\% MG (1:4:5). La proporción de machos en poblaciones de langostinos cultivados en estanques bajo diferentes condiciones ambientales por los general ha sido constante en $50 \% \mathrm{MP}, 40 \% \mathrm{MM}$ y 10\% MG (5:4:1) (Marques y Lombardi, 2011). El mayor cambio proporcional se dio en la fracción de los grandes con los MP y MG mientras que la de los MM se mantuvo constante. En la fracción de los pequeños, los MM y MG representaron el $59 \%$ de la población de machos y en la de los grandes el $91 \%$. Esta observación concuerda con lo establecido en otros estudios donde fue de $60 \%$ y casi $100 \%$ de manera respectiva cuando la separación se hizo en precría (Karplus et al., 1986).

La población compuesta por MP y MM en el grupo de los pequeños mostró un incremento con respecto al de los grandes, mientras que la proporción de MG disminuyó. La proporción de hembras también se redujo de manera apreciable en el grupo de los pequeños sin encontrarse hembras ovígeras. El aumento en la proporción de MM se ha atribuido a la falta de hembras para estimular su transición a MG sexualmente maduros (Tidwell et al., 2015). En poblaciones mixtas con suficientes hembras raramente se aparean por lo que continúan su crecimiento hasta que alcanzan y sobrepasan el tamaño de los MG para convertirse en uno de ellos desplazándolo de su territorio con hembras (Ra'anan y Cohen, 1985). Al existir pocas hembras en la fracción de los pequeños, estos MM crecen aún más hasta alcanzar pesos y longitudes mayores que los del mismo morfo tipo en la de los grandes, como fue observado en este estudio.

Muchos de los MP en el grupo de los pequeños se convirtieron en MM incrementándose su proporción a más del doble (de 19 a 47\%). Estos MM contribuyeron considerablemente al incremento en la producción del grupo de los pequeños. Este cambio compensatorio de MP a MM en el grupo de los pequeños debería reflejarse en un crecimiento compensatorio, pero se enmascara debido a la gran variabilidad de tallas que se incrementa sobre todo cuando están pasando de un morfo tipo al otro (Ra' anan y Cohen, 1985).

Las características de la producción revelaron que el peso de las hembras fluctuó entre 15 y $20 \mathrm{~g}$ con una relación longitud tenazas: cuerpo (0.8) similar en los dos grupos. Los MG en ambas fracciones mostraron el mayor tamaño $(>25 \mathrm{~g})$ en todas las mediciones con respecto a los demás. Se caracterizan por una longitud de tenazas similar al tamaño del cuerpo (1.0) dedicando la mayor parte de su energía al apareamiento en lugar que crecimiento. Los MM ocuparon una posición intermedia en peso (10-20 g) y longitud entre los machos y similar al de las hembras. Están en fase de crecimiento y escasa actividad reproductiva por lo que tienen poca longitud de tenazas con respecto al cuerpo (0.8). Los MP presentaron las menores mediciones en todos los aspectos con pesos inferiores a $10 \mathrm{~g}$ y poca longitud de tenazas: cuerpo (0.7). Esta condición no les impide, sino que les facilita, participar activamente en el apareamiento y la fertilización invirtiendo poca energía en crecimiento. Las relaciones de longitud tenazas: cuerpo de los MP y MM están dentro del rango indicado por Ra'anan y Cohen (1985) de 0.4-0.9 y 0.81.4 , respectivamente, pero fue inferior para los MG (1.4-2.0).

Se ha demostrado la dependencia de los rendimientos del procesamiento de langostinos con el tamaño y el sexo. Es común que el porcentaje del peso de la cola disminuya con el aumento en el tamaño del animal. Los valores promedio en este estudio para las hembras $(49 \%)$ y los machos (48\%) son superiores a los reportados por Smith et al. (2009) con animales más grandes ( $30 \mathrm{~g}$ promedio) los cuales fueron de 38 y $35 \%$, respectivamente. Un porcentaje de 33 a $42 \%$ para las hembras y 26 a $41 \%$ para los machos ha sido 
reportado (Lalrinsanga et al. 2013). El mayor porcentaje en cola de los MP y las hembras podría estar relacionado al menor tamaño y peso de sus tenazas con respecto al cuerpo (2.1 y $2.5 \%$, respectivamente). Ambos son inferiores al de los MM (3.3\%) y los MG (7.0\%) y al promedio de $4.3 \%$ para todos los machos. Una relación muy similar entre el peso de las tenazas y el cuerpo fue reportada por Smith et al. (2009) con valores de $2.8 \%$ para las hembras y $4.3 \%$ para los machos en animales de $30 \mathrm{~g}$ promedio. En este estudio solo los MG tuvieron un peso superior en cabeza y tenazas con respecto a las hembras, mientras que los MM tuvieron un peso similar y el de los MP fue inferior. En el estudio de Lalrinsanga et al. (2013) se reportó que el tamaño y peso de la cabeza y las tenazas de los MG dentro de 41-50 g se incrementan considerablemente con respecto a las hembras, mientras se hace más angosto el abdomen y se reduce el porcentaje de carne en la cola.

\section{Conclusiones}

- Los resultados preliminares del estudio muestran, bajo las condiciones del cultivo comercial en fases, la inexistencia de crecimiento compensatorio de la precría en alta densidad al desarrollo en densidad media de siembra y al engorde con la baja densidad de siembra, tradicionalmente utilizada de manera convencional en el país.

- El cultivo de langostinos en alta densidad en precría parece ser la mejor estrategia de producción para aumentar la productividad de los cultivos pasándolos directamente al engorde definitivo obviando la fase de desarrollo. Tiene como ventajas la alta sobrevivencia, el aumento en el tamaño de siembra, la reducción de la susceptibilidad del animal a las nuevas condiciones de cultivo y el cambio compensatorio.

- Al separar los langostinos por tamaño para sembrarlos en el engorde se notó crecimiento compensatorio en los perte- necientes a la fracción de los grandes dentro de un amplio rango de variabilidad de tallas. El cambio compensatorio tuvo mayor influencia en los rendimientos de producción. Al separar los que crecen más rápido de los demás, se quita la supresión del crecimiento sobre todo a los MP, los cuales pueden desarrollar todo su potencial de crecer. Se suspende la tasa de crecimiento diferencial socialmente inducida que contribuye a la disparidad de tallas en la población a través del tiempo.

- Los MP son los más favorecidos sin la presencia de los grandes por varias razones de comportamiento social alcanzando un cambio compensatorio para llegar a ocupar el nicho vacante dejado por los machos más grandes.

- El manejo adecuado del cambio compensatorio se facilita con las cosechas totales, vaciando el estanque y separando todos los grandes mediante la implementación del cultivo en fases.

- Una de las principales ventajas del cultivo en fases es que se aumenta la productividad al poder realizarse mayor cantidad de ciclos al año y el tamaño a cosecha de los MP por medio de la separación de tallas.

\section{Literatura Citada}

1. Ahmed N. 2013. On-farm feed management practices for giant river prawn (Macrobrachium rosenbergii) farming in southwest Bangladesh. FAO Fisheries and Aquaculture. Technical Paper No. 583: 269-301.

2. Akter S, Mosharraf M, Banerjee S, Kumar B, Zaman F. 2014. Farming of giant freshwater prawn (Macrobrachium rosenbergii) in Bagerhat, Bangladesh. J Fisheries 2: 187-194. doi: dx.doi.org $/ 10.17017 /$ jfish

3. Al-Mohsen I. 2009. Macrobrachium rosenbergii (de Man 1879): the antennal gland and the role of pheromones in 
mating behaviour. PhD Thesis. Stirling: University of Stirling. 253 p.

4. Alston D, Sampaio C. 2000. Nursery systems and management. In: New WM, Valenti W (eds). Freshwater prawn culture: the farming of Macrobrachium rosenbergii. London: BlackwellScience. p 112-122.

5. Boyd C. 2017. La cal desempeña un papel crcucial en el manejo de estanques acuícolas. Global Aquaculture Alliance. [Internet]. Available in: https://www.aquaculturealliance.org/advocate/la-caldesempena-un-papel-crucial...

6. Boyd C, Zimmermann S. 2000. Growout systems: water quality and soil management. In: New WM, Valenti W (eds). Freshwater prawn culture: the farming of Macrobrachium rosenbergii. London: Blackwell-Science. p 221-238.

7. D'Abramo L, New M. 2000. Nutrition, feeds and feeding. In: New WM, Valenti W(eds). Freshwater prawn culture: the farming of Macrobrachium rosenber-gii. London: Blackwell-Science. p 203-216.

8. Daniels W, D'Abramo L. 1994. Pond production characteristics of freshwater prawns Macrobrachium rosenbergii as influenced by the stocking of size-graded populations of juveniles. Aquaculture 122:33-45. doi: 10.1016/0044-8486(94)90331-X

9. Gao X, Zhou Y, Zhu X, Tang H, Li X, Jiang Q, Wei W, Zhang X. 2021. Enterobacter cloacae: A probable etiological agent associated with slow growth in the giant freshwater prawn Macrobrachium rosenbergii. Aquacul-ture 530: 735826. doi: 10.1016/j.aquacul-ture

10. Holthuis L. 2000. Nomenclature and taxonomy. In: New WM, Valenti W (eds). Freshwater prawn culture: the farming of Macrobrachium rosenbergii. London: Blackwell-Science. p 12-16.

11. Jia P, Zhi L, Zi Y, Martínez L, Osorio D, Torres M. 1988. Cultivo intensivo del camarón de agua dulce Macrobrachium rosenbergii (De Man). Act Cient Tecn Inderena 1: 45-55.
12. Johnson S, Bueno S. 2000. Health management. In: New WM, Valenti W (eds). Freshwater prawn culture: the farming of Macrobrachium rosenbergii. London: Blackwell-Science. p 239-253.

13. Karplus I, Malecha S, Sagi A. 2000. The biology and management of size variation. In: New MB, Valenti W (eds). Freshwater prawn culture: the farming of Macrobrachium rosenbergii. London:Blackwell Science. p 259-282.

14. Karplus I, Hulata G, Wohlfarth G, Halevy A. 1986. The effect of sizegrading juvenile Macrobrachium rosenbergii prior to stocking on their population structure and production in polyculture: I. Dividing the population into two fractions. Aquaculture 56: 257-270. doi: 10.1016/0044-8486(87)90313-9

15. Lalrinsanga P, Pillai B, Patra G, Mohanty S, Kumari N, Ranjan R, Sahu S, et al. 2013. Yield characteristics and morphometric relationships of giant freshwater prawn, Macrobrachium rosenbergii (de Man, 1879). Aquacult Int 22: 1053-1066. doi: 10.1007/ s10499-013-9726-6

16. Madhusoodana K, Ranjeet K. 2005. Multiple stocking for enhancing survival, production and marketable yield structure of Macrobrachium rosenbergii (de Man). Indian J Fish 52: 37-46,

17. Malecha S. 1988. Recent advances in the production of the freshwater prawn Macrobrachium rosenbergii. In: Proc $1^{\text {st }}$ Congress of Aquaculture International. Vancouver: British Columbia Corporation.

18. Malecha SR, Sandifer PA, Cotsapas L. 1989. Potential application to Brazil of recent advances in the culture of marine shrimps and freshwater prawns. In: Brazilian Symposium on prawn culture. Brazil.

19. Marques L, Lombardi J. 2011. Compensatory growth of Malaysian prawn reared at high densities during the nursery phase. Rev Bras Zootecn 40: 701-707. soi: 10.1590/S1516-35982011000400001 
20. Marques H, Moraes-Valenti P. 2012. Current status and prospects of farming the giant river prawn (Macrobrachium rosenbergii De Man 1879) and the Amazon River prawn Macrobrachium amazonicum (Heller 1862) in Brasil. Aquac Res 43: 984-992. doi: 10.1111/ j.1365-2109.2011.03032.x

21. Nair M, Salin K. 2012. Current status and prospects of farming the giant river prawn Macrobrachium rosenbergii (De Man) and the monsoon river prawn Macrobrachium malcolmsonii. Aquac Res 43: 999-1014. doi: 10.1111/j.13652109.2011.03074.x

22. Negrini C, Silva C, Bittencourt A, Frozza A, Ortiz R, Cupertino E. 2017. Stocking density for freswater prawn Macrobrachium rosenbergii (Decapoda, Palaemonidae) in biofloc system. Lat Am J Aquat Res 45: 891-899. doi: 10.3856/vol45.

23. New M. 2000a. Commercial freshwater prawn farming around the world. In New M, Valenti W (eds). Freshwater prawn culture: the farming of Macrobrachium rosenbergii. London: Blackwell Science. p 291-323.

24. New M. 2000b. History and global status of freshwater prawn farming. In: New M, Valenti W (eds). Freshwater prawn culture: the farming of Macrobrachium rosenbergii. London: Blackwell Science. p 1-11.

25. New M, D'Abramo L, Valenti W, Singholka S. 2000. Sustainability of freshwater prawn culture. In: New M, Valenti W (eds). Freshwater prawn culture: the farming of Macrobrachium rosenbergii. London: Blackwell Science. p 429-433.

26. New M, Kutty M. 2010. Commercial freshwater prawn farming and enhancement around the world. In: New M, Valenti W, Tidwell J, D’Abramo L, Kutty $M$ (eds). Freshwater prawn biology and farming. Oxfrod: Wiley-Blackwell. p346-399.

27. Prates E, Holanda M, Waisielesky W. 2020. Evaluating compensatory growth in Pacific white shrimp in a biofloc system. Global Aquaculture Advocate. [Internet]. Available in: https:// www.aquaculturealliance.org/advocate/ evaluating-compensatory-growth..

28. Ra'anan Z, Cohen D. 1985. Ontogeny of social structure and population dynamics in the giant freshwater prawn Macrobrachium rosenbergii (De Man). In: Wenner A, Schram F (eds). Crustacean Issues, Vol. 2: Crustacean growth. Rotterdam: AA Balkema. p 277-311.

29. Rhodes R. 2000. Economics and business management. In: New M, Valenti W (eds). Freshwater prawn culture: the farming of Macrobrachium rosenbergii. London: Blackwell Science. p 369-392.

30. Samad M, Kumar A, Rezoanul M. 2016. Growth and economic performances of Macrobrachium rosenbergii (De Man, 1879) culture in South-West coastal region of Bangladesh. J Environ Sci Natural Resources 9: 53-57. doi: 10.3329/jesnr.v9i1.30291

31. Shivakumar M, Naveenkumar B, Shivananda $M$, Ramachandra $N$ Vasudevappa C. 2012. Studies on effect of claw-ablation on growth and survival of Macrobrachhium rosenbergii (de Man). Int J Sci Environ Technol 1: 491-498.

32. Singh S. 2011. Freshwater prawn Macrobrachium rosenbergii (De Man, 1879) (Crustacea: Decapoda) aquaculture in Fiji: Improving culture stock quality. MSc Thesis. Fiji: University of the South Pacific. 89 p.

33. Smith T, Waltz W, Sandifer P. 2009. Processing yields for Malaysian prawns and the implications. J World Aquacult Soc 11: 557-569. doi: 10.1111/j.17497345.1980.tb00150.x

34. Tidwell J. 2012. Current status and prospects of farming the giant river prawn (Macrobrachoum rosenbergii De Man 1879) in the United States. Aquac Res 43: 1023-1028. doi: 10.1111/ j.1365-2109.2012.03130.x

35. Tidwell J, D'Abramo L. 2010. Growout systems - culture in temperate zones. In: New M, Valenti W, Tidwell J, 
D’Abramo L, Kutty M (eds). Freshwater prawn biology and farming. Oxford: Wiley-Blackwell. p 180-194.

36. Tidwell J, D'Abramo L. 2000. Growout sistems - Culture in temperate zones. In: New M, Valenti W (eds). Freshwater prawn culture: the farming of Macrobrachium rosenbergii. London: Blackwell Science. p 177-186.

37. Tidwell J, Coyle S, VanArnum A, Weibel, C. 2003. The effect of size grading and length of nursery period on growth and population structure of freshwater prawns stocked in temperature zone ponds with added substrates. Aquaculture 218: 209-218. doi: 10.1016/ S0044-8486(02)00341-1

38. Tidwell JH, Odeyemi OO, Coyle SD, Schneider KJ, Bright LA. 2014. Production comparison of three genetic strains of fresh water prawn, Macrobrachium rosenbergii, raised under two pond management technologies. J World Aquacult Soc 45: 595-603. doi: 10.1111/ jwas. 12148

39. Tidwell J, D'Abramo L, Coyle S, Bright L, Shultz C, Kupchinsky Z, Schwartz P, Upstrom C. 2015. Production characteristics of all-male and mixed-sex giant river prawn, Macrobrachium rosenbergii, grown in earthen ponds in Ketucky and Mississippi, USA. J World Aquac Soc 46: 139-140. doi: 10.1111/jwas. 12174
40. Tidwell J, D'Abramo L, Coyle S, Yasharian D. 2005. Overview of recent research and development in temperate culture of the freshwater prawn (Macrobrachium rosenbergii De Man) in the South Central United States. Aquac Res 36: 264-277. doi: $10.1111 / \mathrm{j} .1365-$ 2109.2005.01241.x

41. Valenti W, New M. 2000. Grow-out systems - monoculture. In: New M, Valenti W (eds). Freshwater prawn culture: the farming of Macrobrachium rosenbergii. London: Blackwell Science. p 157-176.

42. Valverde J, Varela A. 2020. Efecto de la densidad de siembra en la productividad y rentabilidad del langostino Macrobrachium rosenbergii en la fase de engorde en estanques, Costa Rica. Rev Inv Vet Perú 31: e18174. soi: 10.15381/rivep.v31i3.18174

43. Valverde-Moya J, Alfaro-Montoya J. 2015. Crecimiento compensatorio y producción en las fases de precría, desarrollo y engorde comercial del camarón blanco Litopenaeus vannamei, en Costa Rica. Rev Mar Cost 7: 99-115.

44. Varela-Mejías A, Valverde-Moya J. 2018. Determinación de la causa de mortalidad en un vivero del langostino gigante de agua dulce Macrobrachium rosenbergii en Costa Rica: análisis de caso. Rev Inv Vet Perú 29: 666-675. doi: 10.15381/rivep.v20i2.14522 